\title{
Comparative Statics in an Ordinal Theory of Choice under Risk
}

DOI:

10.1016/j.mathsocsci.2019.07.004

\section{Document Version}

Accepted author manuscript

Link to publication record in Manchester Research Explorer

\section{Citation for published version (APA):}

Chiu, W-J. (2019). Comparative Statics in an Ordinal Theory of Choice under Risk. Mathematical social sciences, 101, 113-123. https://doi.org/10.1016/j.mathsocsci.2019.07.004

\section{Published in:}

Mathematical social sciences

\section{Citing this paper}

Please note that where the full-text provided on Manchester Research Explorer is the Author Accepted Manuscript or Proof version this may differ from the final Published version. If citing, it is advised that you check and use the publisher's definitive version.

\section{General rights}

Copyright and moral rights for the publications made accessible in the Research Explorer are retained by the authors and/or other copyright owners and it is a condition of accessing publications that users recognise and abide by the legal requirements associated with these rights.

\section{Takedown policy}

If you believe that this document breaches copyright please refer to the University of Manchester's Takedown Procedures [http://man.ac.uk/04Y6Bo] or contact uml.scholarlycommunications@manchester.ac.uk providing relevant details, so we can investigate your claim.

\section{OPEN ACCESS}




\title{
Comparative Statics in an Ordinal Theory of Choice under Risk
}

\author{
W. Henry Chiu \\ Economics, School of Social Sciences \\ The University of Manchester \\ Manchester, M13 9PL, U.K.
}

July 9, 2019

\begin{abstract}
This paper considers the effects of changes in risk on optimal decisions under risk in an analytical framework that is capable of replicating and extending important results obtained in the EU framework and is yet far more descriptive and no less tractable at the same time. We show that a version of the general framework can be used to establish in the class of problems considered by Rothschild and Stiglitz (1971) and Machina (1989) the equivalence between behavioral assumptions and the characteristics of the utility function, which are in turn necessary and sufficient for comparative statics results.
\end{abstract}

Key Words: utility-representable preferences, ordinal utility, portfolio choice, comparative statics, prudence, precautionary saving, labor supply.

JEL Classification: D81. 


\section{Introduction}

A large part of the economics of risk and uncertainty is justly concerned with how the introduction of risk, or more generally changes in risk, affect individuals' economic behavior. The lasting popularity of the Expected Utility (EU) model is in no small part attributable to the sharp comparative statics results that can be generated in the framework under conditions on the von Neumann-Morgenstern (VNM) utility function that in many cases have plausible behavioral interpretations. For a large class of problems, Rothschild and Stiglitz (1971) provide a sufficient condition on the VNM utility function for ascertaining the effect of an increase in risk (in the sense defined in Rothschild and Stiglitz (1970)) of some underlying economic variable on the optimal choice of a control variable. The condition in its general form however has no obvious behavioral interpretation. ${ }^{1}$ In the more specific setting of a two-asset portfolio choice problem, Cheng, Magill, and Shafer (1987) Hadar and Seo (1990) respectively identify the conditions on the VNM utility function under which the effects on the optimal portfolio choice can be ascertained when the return of one of the assets deteriorates in the senses of first-degree and second-degree stochastic dominance. Chiu, Eeckhout, and Rey (2012) generalize these results by establishing the effects of a deterioration in the return of one of the assets in the sense of an $N$ th-degree risk increase (Ekern (1980)) ${ }^{2}$ and further offer a behavioral interpretation for the condition on the VNM utility function sufficient for the result.

Violations of the independence axiom that underpins the EU model such as the Allais paradox and the common ratio effect are, however, well-known by the 1960s. In response, a number of "non-expected utility theories" have been proposed, the most successful among which is what becomes known as Rank-Dependent Expected Utility (RDEU) theory first put forward by Quiggin (1982). 3 To account for other patterns of experimental evidence they uncover as well as those similar to the Allais paradox, Kahneman and Tversky (1979) propose Prospect Theory that allows for preferences to be reference-dependent. What is termed Cumulative Prospect Theory was later proposed and axiomatized to allow for reference-dependent preferences while retaining both

\footnotetext{
${ }^{1}$ The class of problems considered by Rothschild and Stiglitz (1971) takes the form of choosing $q$ to maximize $E u(\tilde{x}, q)$. They show that if $u_{q x x}(\cdot, \cdot)<0$, then a mean-preserving increase in risk in $\tilde{x}$ lowers the optimal choice of $q$. As can be seen in their analysis of more specific problems, possible behavioral interpretations of the condition $u_{q x x}(\cdot, \cdot)<0$ depend on the specification of the relationship between $\tilde{x}$ and $q$.

${ }^{2}$ The notion of an Nth-degree risk increase nests that of a downside risk increase defined by Menezes, Geiss and Tressler (1980) as well as those of a first-degree stochastic dominant deterioration and Rothschild and Stiglitz's (1970) notion of increasing risk.

${ }^{3}$ See Starmer (2000) for an evaluation of available experimental evidence that appears to favor rank-dependent models over other non-EU models, esp. those assuming "betweenness" and "fanning out" as a weaker replacement for linearity in probabilities.
} 
monotonicity and transitivity, which Prospect Theory was shown not to always obey simultaneously. ${ }^{4}$ While these models allow for non-linearity in probabilities, they all retain a form of partial event-separability to permit an explicit specification of the relationship between the utility of a distribution and the utilities of its possible outcomes. ${ }^{5}$ Machina (1982a, b), Machina and Neilson (1987), Machina (1989), and Wang (1993), on the other hand, show that, without the independence axiom or a weaker replacement for it, most "EU analysis" can be carried out with the "local utility function" (which is the probability derivative of the preference functional) taking the place of the von Neumann-Morgenstern (VNM) utility function as long as a differentiability condition on the preference-representing functional is assumed.

These impressive theoretical advances notwithstanding, while its dominance is somewhat diminished, the EU model continues to be the most widely used analytical framework for not just empirical but also theoretical research in the economics of uncertainty. What explains its lasting popularity in the presence of patently more descriptive alternatives, as many researchers observe, is the fact that none of the alternative models is as simple and easy to use as the EU model. The greater complexity of these alternative models is furthermore seen as inevitable as they can all be understood as attempts at a generalized version of "EU analysis" - analysis of choice under risk based on the characterizations of preference regularities in terms of an analogue of the VNM utility function - where a weaker replacement for the independence axiom necessarily imply a more complex preference functional. ${ }^{6}$ What underlies this seemingly inevitable modelling trade-off is the implicit and nearly consensual belief that a model without a degree of cardinalization necessary for EU analysis ${ }^{7}$ is incapable of replicating a version of the elegant characterizations of preferences and the multitudes of intuitively appealing results on optimal decisions derived in the EU framework, or worse, it would be a model void of empirical contents. While this is understandable in view of the fact that Bernoulli's original EU hypothesis was a response to the observed inadequacy of the even

\footnotetext{
${ }^{4}$ An exposition of this can be found in Starmer (2000).

${ }^{5}$ The independence axiom, which implies and is implied by full event separability, entails, among other things, the additive separability of the preference functional in the utilities of possible outcomes.

${ }^{6}$ The "local utility" approach taken by Machina (1982a) and Wang (1993) where no weaker replacement for the Independence axiom is assumed permits the generalization of a number of general regularities obtained in the EU framework and has been used to derive important properties in non-EU models (e.g. Chew, Karni and Safra (1987)). It is however far more technically demanding and less simple and easy to use than the approach taken in this paper, especially in more complex settings such as ones with correlated endogenous and background risks.

${ }^{7}$ The EU or any of the existing non-EU preference functionals of course need not be seen as cardinal as any monotone increasing transformation of it still represents the same preferences over distributions. But the VNM utility function and its analogues in non-EU models including the local utility function in Machina's (1982a) and the value function in Kahneman and Tversky's (1979) prospect theory are necessarily cardinal.
} 
earlier hypothesis that individuals choose among lotteries on the basis of their expected values, it should seem peculiar in the wider context of consumer choice theory where it was widely recognized by the 1940s that the assumption of cardinal utility is not just unnecessary but undesirable. What is more, even the arguably most descriptive to date of the non-EU models, namely cumulative prospect theory, with its much more unwieldy preference functional, is not without experimental evidence challenging its theoretical underpinning: All rank-dependent models, including cumulative prospect theory, imply "ordinal independence", systematic violations of which have been observed in experiments (Wu (1994)). Since ordinal independence is a form of partial "event separability" as explained in Machina (2009), available experimental evidence is seen to be inconsistent not just with preference functionals linear in probabilities but also with those assuming weaker forms of event separability, the very property that makes possible the derivation of an analogue of the VNM utility function in non-EU models.

This paper shows that a meaningful ordinal theory of choice under risk capable of replicating and extending important results derived in the EU framework is not just feasible but due to its more general and yet simpler nature, it can be more descriptive and no less tractable at the same time. Furthermore, being analytically more akin to standard consumer theory, the analysis of decision under risk in this framework employs little more than elementary mathematical techniques and, even in fairly general cases is amenable to simple graphical illustration. The key to this more general and yet simpler analytical framework is to dispense with EU analysis altogether and use instead a parameter-based representation of changes in probability distribution resulting from decisions under risk. More specifically, while we assume, as do existing models, that preferences over probability distributions are representable by a utility function, unlike existing models, we make no further assumption to cardinalize the preference-representing utility function in any way. In particular, there is no assumption that implies any form of event-separability and thus there is no explicit specification of how the utility of a distribution relates to the utility of each of the distribution's possible outcomes obtained with certainty. Neither is there any assumption of differentiability on the preference-representing utility function (which requires a cardinalization) to derive the "local utility". As a result, there is no analogue of the VNM utility function. For characterizing behavioral assumptions, we use instead an ordinal utility function of decision parameters enabled by the parameter-based representation of distributional changes. Focusing in this paper on the effects of changes in risk on optimal decisions under risk, we show that a version of the general framework 
can be used to establish in the class of problems considered by Rothschild and Stiglitz (1971) and Machina (1989) the equivalence between behavioral assumptions and the characteristics of the preference-representing utility function, which are in turn necessary and sufficient for comparative statics results. That is, we show that not only can existing comparative statics results be extended to an analytical framework that require no assumption of a differentiability condition or a version of the independence axiom but their necessary and sufficient conditions can also be characterized in terms, not of the VNM or local utility functions whose behavioral interpretations may not be immediately clear, but of behavioral assumptions on the preferences themselves.

As the analytical framework assumes no event-separability of any kind, it is consistent with all observed patterns of behavior, including those reported in Wu (1994) that violate "ordinal independence". As shown in Chiu (2018a), in this framework a behavioral hypothesis can instead be formulated that accounts for the full range of putatively paradoxical behavioral patterns including simultaneous gambling and insurance, the common ratio effect, Kahneman and Tversky's (1979) reflection effect, and preference reversal. Chiu (2018b) further shows that the analytical framework can be extended to a setting with subjective uncertainty where preference conditions on attitudes towards ambiguity can be identified that account for not just the Ellsberg paradox but also behavioral patterns suggested by Machina $(2009,2013)$ to be inconsistent with the predictions of major existing models of ambiguity aversion. In the context of our theoretical framework assuming only utility-representable preferences, far from being void of empirical contents, the behavioral hypotheses in terms of preferences over distributions, not conditions on an analogue of the VNM utility function, are more precise and are ready to be more precisely tested in experiments.

The rest of the paper is organized as follows. Section 2 sets out notions of a risk deterioration and key behavioral assumptions. Section 3 develops the analytical framework for decision-making under risk assuming only utility-representable preferences and discusses the comparative statics results that can be obtained. Section 4 concludes.

\section{Changes in Risk and Behavioral Regularities}

Risky prospects or risks are represented by random variables $\tilde{x}$, $\tilde{y}$, etc., that take values in an interval $[a, b] \subset R$ and their cumulative distribution functions are denoted by $F_{\tilde{x}}, F_{\tilde{y}}$, etc. We use the notion of an $N$ th-degree risk increase introduced by Ekern (1980) to summarize existing notions 
of a risk deterioration. For a distribution function $F_{\tilde{x}}(x)$, defining $F_{\tilde{x}}^{(1)}(x)=F_{\tilde{x}}(x)$ and

$$
F_{\tilde{x}}^{(n+1)}(x)=\int_{a}^{x} F_{\tilde{x}}^{(n)}(y) d y \text { for all } x \in[a, b] \text { and all } n \in\{1,2, \ldots\},
$$

the notion of an $N$ th-degree risk increase is defined as follows.

Definition $1 F_{\tilde{x}^{\prime}}$ is an $N$ th-degree risk increase of $F_{\tilde{x}}$ if $F_{\tilde{x}}^{(N)}(x) \leq F_{\tilde{x}^{\prime}}^{(N)}(x)$ for all $x \in[a, b]$ where the inequality is strict for some $x$ and $F_{\tilde{x}}^{(n)}(b)=F_{\tilde{x}^{\prime}}^{(n)}(b)$ for $n=2, \ldots, N$.

As pointed out by Ekern (1980), the condition $F_{\tilde{x}}^{(n)}(b)=F_{\tilde{x}^{\prime}}^{(n)}(b)$ for $n=2, \ldots, N$ means the first $(N-1)$ moments of $\tilde{x}$ and $\tilde{x}^{\prime}$ are equal. ${ }^{8}$ Thus $F_{\tilde{x}^{\prime}}$ being a first-degree risk increase of $F_{\tilde{x}}$ is clearly the same as $F_{\tilde{x}}$ first-degree stochastically dominating $F_{\tilde{x}^{\prime}}$ and a second-degree risk increase is equivalent to Rothschild and Stiglitz's (1970) notion of increasing risk termed "mean-preserving increase in risk" by Machina and Pratt (1997). ${ }^{9}$ The following important properties of the concept are proved in Rothschild and Stiglitz (1970).

Lemma 1 (Rothschild and Stiglitz (1970)) The following statements are equivalent.

(i) $F_{\tilde{x}^{\prime}}$ is a mean-preserving increase in risk (MPIR) of $F_{\tilde{x}}$;

(ii) $E u\left(\tilde{x}^{\prime}\right) \leq E u(\tilde{x})$ for all concave function $u$;

(iii) There exists $\tilde{\epsilon}$ such that $F_{\tilde{x}^{\prime}}=F_{\tilde{x}+\tilde{\epsilon}}$ and $E[\tilde{\epsilon} \mid \tilde{x}]=0$.

A third-degree risk increase, on the other hand, is equivalent to a downside risk increase as defined by Menezes, Geiss and Tressler (1980), which corresponds to a dispersion transfer from higher to lower wealth levels and implies a decrease in skewness as measured by the third central moment. A fourth-degree risk increase is further equivalent to what Menezes and Wang (2005) define to be an increase in outer risk which corresponds to a dispersion transfer from the center of a distribution to its tails while maintaining its mean, variance and skewness (i.e., third central moment). The following property of an $N$ th-degree risk increase in the EU framework can be proved analogously to the main result in Ekern (1980).

\footnotetext{
${ }^{8}$ The notion of an $N$ th-degree risk increase is closely related to that of $N$ th-degree stochastic dominance: $F_{\tilde{x}}$ is said to $N$ th-degree stochastically dominate $F_{\tilde{x}^{\prime}}$ if $F_{\tilde{x}}^{(N)}(x) \leq F_{\tilde{x}^{\prime}}^{(N)}(x)$ for all $x \in[a, b]$ where the inequality is strict for some $x$. All the preference conditions defined in what follows in terms of an $N$ th-degree risk increase can be analogously defined in terms of $N$ th-degree stochastic dominance. The characterizations of preference conditions so defined in the EU framework as well as in our general parameter-based framework are completely analogous to what to be presented in the sequel.

${ }^{9}$ The conditions $F_{\tilde{x}}^{(2)}(x) \leq F_{\tilde{x}^{\prime}}^{(2)}(x)$ for all $x \in[a, b]$ where the inequality is strict for some $x$ and $F_{\tilde{x}}^{(2)}(b)=F_{\tilde{x}^{\prime}}^{(2)}(b)$ correspond to the "integral conditions" for Rothschild and Stiglitz's (1970) notion of increasing risk.
} 
Lemma $2 E u\left(\tilde{x}^{\prime}\right) \leq(\geq) E u(\tilde{x})$ for $F_{\tilde{x}^{\prime}}$ being an $N$ th-degree risk increase of $F_{\tilde{x}}$, if and only if $(-1)^{N} u_{(N)} \leq(\geq) 0$.

Denoting by $F_{\tilde{x}_{1}, \ldots, \tilde{x}_{n}}$ the cumulative distribution of the random vector $\left(\tilde{x}_{1}, \ldots, \tilde{x}_{n}\right)$ and by $D[a, b]^{n}$ the set of cumulative distribution functions over $[a, b]^{n}$, an individual preference ordering on $D[a, b]^{n}$, denoted by $\succsim^{(n)}$, is a complete and transitive binary relation that is also assumed to be "continuous in distribution" 10 and is thus, by Debreu (1964), representable by a continuous real-valued preference functional $V^{(n)}(\cdot)$ on $D[a, b]^{n}$. As we will be focusing primarily on the case of $n=2$ in this paper, for notation simplicity, $\succsim^{(2)}$ and $V^{(2)}(\cdot)$ will be abbreviated as $\succsim$ and $V(\cdot)$ respectively. The behavioral conditions to follow are given in terms of preferences over bivariate distributions with one of the random variables being degenerate as it is adequate for the purpose of this paper although they can all be analogously given in terms of general bivariate distributions. Accordingly we will denote by $F_{\tilde{x}, y}$ the cumulative distribution function of the random vector $(\tilde{x}, \tilde{y})$ where $\tilde{y} \equiv y \in[a, b]$.

Definition 2 (Mean-Monotonicity) $\succsim$ exhibits mean-monotonicity if $F_{\tilde{x}+\Delta x, y} \succsim F_{\tilde{x}, y}$ and $F_{\tilde{x}, y+\Delta y} \succsim F_{\tilde{x}, y}$ for all $\tilde{x}, y \in[a, b], \Delta x>0$ and $\Delta y>0$ such that $F_{\tilde{x}+\Delta x, y+\Delta y} \in D[a, b]^{2}$.

Mean-monotonicity thus means that an upward shift of a (bivariate) distribution (in the direction of either variable) is preferred. ${ }^{11}$

Definition 3 (Risk Aversion) $\succsim$ is risk averse if $F_{\tilde{x}, y} \succsim F_{\tilde{x}^{\prime}, y}$ for all $y \in[a, b]$ and $\tilde{x}$ and $\tilde{x}^{\prime}$ such that $F_{\tilde{x}^{\prime}}$ is an MPIR of $F_{\tilde{x}}$.

That is, risk aversion in this paper simply means aversion to an MPIR (in the first attribute). ${ }^{12}$ A preference condition closely related to risk aversion is what Machina and Neilson (1987) and Dekel (1989) term "diversification". ${ }^{13}$ The strict version of this condition is made by Machina and Neilson (1987) so that the choice of $q$ maximizing the utility of $F_{\tilde{w}+q \tilde{x}}$ is unique and characterized

\footnotetext{
${ }^{10}$ That is, the sets $\left\{G \in D[a, b]^{n}: F \succsim^{(n)} G\right\}$ and $\left\{G \in D[a, b]^{n}: G \succsim^{(n)} F\right\}$ are closed in the topology of convergence in distribution.

${ }^{11}$ This is clearly weaker than the more commonly used notion of monotonicity, which says that a first-degree stochastically dominating distribution of the first attribute is preferred. Note however that in the EU framework both notions imply and are implied by a positive first partial derivative of the VNM utility function with respect to the first attribute.

${ }^{12}$ For a discussion on other notions of risk aversion and their characterizations in the EU and non-EU models, see Cohen (1995).

${ }^{13}$ The definition of diversification by Machina and Neilson (1987) and Dekel (1989) and its earlier variants used in Machina (1982a, 1982b) are all meant to be generalizations of Tobin's (1958) original concept of diversification that is characterized by upward-sloping convex mean-variance indifference curves (Machina (1982a, p.299, 1982b, p.1073), Machina and Neilson (1987, p.1145) and Dekel (1989, pp.163-4)).
} 
by the first-order condition and it means that "diversification pays" in a multiple-asset portfolio choice problem. For a condition under which the optimal choice in our setting is still characterized by the first-order conditions, we shall assume a somewhat stronger preference condition, which we term "co-bivariate-diversification".

\section{Definition 4}

(i) $\succsim$ exhibits (strict) co-bivariate-diversification (C-diversification) if $\succsim$ over $\left\{F_{\tilde{w}+q \tilde{x}, \Gamma+\beta q}\right\}_{q}$ is (strict) quasi-concave in $q$ for all random variables $\tilde{w}$ and $\tilde{x}$ and $(\Gamma, \beta) \in R^{2}$.

(ii) $\succsim$ exhibits (strict) diversification if $\succsim$ over $\left\{F_{\tilde{w}+q \tilde{x}, y}\right\}_{q}$ is (strict) quasi-concave in $q$ for all random variables $\tilde{w}$ and $\tilde{x}$ and $y \in[a, b]$.

In the EU framework, both diversification and risk aversion imply and are implied by the concavity of the VNM utility function (Dekel (1989)). Without assuming EU maximization, Dekel (1989) and Chateauneuf and Lakhnati (2007) show that diversification implies risk aversion but not vice versa. Being a stronger condition than diversification, C-diversification thus also implies risk aversion.

We next introduce the condition on the preferences over distributions that in the context of a two-period consumption/saving problem will be shown to be necessary and sufficient for a precautionary saving motive.

\section{Definition 5 (prudence)}

$\succsim$ exhibits $N$ th-degree prudence if $F_{\tilde{w}+\pi, y-\delta} \sim F_{\tilde{w}, y}$ implies $F_{\tilde{w}^{\prime}+\pi, y-\delta} \succsim F_{\tilde{w}^{\prime}, y}$ for all $\tilde{w}, \tilde{w}^{\prime}, y \in$ $[a, b], \pi \in R$ and $\delta \in R$ such that $F_{\tilde{w}^{\prime}}$ is an $N$ th-degree risk increase of $F_{\tilde{w}}$ and $F_{\tilde{w}+\pi, y-\delta}, F_{\tilde{w}^{\prime}+\pi, y-\delta}$ $\in D[a, b]^{2}$.

That is, an individual exhibits $N$ th-degree prudence if she dislikes a reduction in the second attribute less when the background risk in the first attribute worsens in the sense of an $N$ th-degree risk increase. In a simple two-period EU-based consumption-saving model with a time-separable VNM utility function, Leland (1969) first shows that a precautionary saving motive is implied by a positive third derivative of the VNM utility function, which thanks to the work of Kimball (1990), has become known as "prudence". The characterization of our notion of prudence in the EU framework is given in the Appendix where we show that, under the conditions assumed in Leland (1968) and Kimball (1990), this notion of prudence implies and is implied by a positive third derivative of the VNM utility function. 
Denoting the conditional distribution of $\tilde{\epsilon}$ on $\tilde{w}$ by $F_{\tilde{\epsilon} \mid \tilde{w}}$, we now introduce the preference condition of judicious risk appetite.

\section{Definition 6 (Judicious Risk Appetite)}

(i) $\succsim$ exhibits $N$ th-degree judicious appetite for risk if $F_{\tilde{w}+\tilde{\epsilon}, y} \sim F_{\tilde{w}+\pi+\alpha \tilde{\epsilon}, y}$ implies $F_{\tilde{w}+\tilde{\epsilon}^{\prime}, y} \succsim$ $F_{\tilde{w}+\pi+\alpha \tilde{\epsilon}^{\prime}, y}$ for all $\tilde{w}, \tilde{\epsilon}, \tilde{\epsilon}^{\prime}, y \in[a, b], \pi \in R$ and $\alpha>1$ such that $E[\tilde{\epsilon}]=0, F_{\tilde{\epsilon}^{\prime} \mid \tilde{w}}$ is an $N$ th-degree risk increase of $F_{\tilde{\epsilon} \mid \tilde{w}}$ and $F_{\tilde{w}+\pi+\alpha \tilde{\epsilon}, y}, F_{\tilde{w}+\pi+\alpha \tilde{\epsilon}^{\prime}, y} \in D[a, b]^{2}$.

(ii) $\succeq$ exhibits $N$ th-degree judicious appetite for aggravated risk if $F_{\tilde{w}+\tilde{\epsilon}, y} \sim F_{\tilde{w}+\pi+\alpha \tilde{\epsilon}, y-\delta}$ implies $F_{\tilde{w}+\tilde{\epsilon}^{\prime}, y} \succsim F_{\tilde{w}+\pi+\alpha \tilde{\epsilon}^{\prime}, y-\delta}$ for all $\tilde{w}, \tilde{\epsilon}, \tilde{\epsilon}^{\prime}, y \in[a, b], \pi \in R, \delta \geq 0$ and $\alpha>1$ such that $E[\tilde{\epsilon}]=0$, $F_{\tilde{\epsilon}^{\prime} \mid \tilde{w}}$ is an Nth-degree risk increase of $F_{\tilde{\epsilon} \mid \tilde{w}}$ and $F_{\tilde{w}+\pi+\alpha \tilde{\epsilon}, y-\delta}, F_{\tilde{w}+\pi+\alpha \tilde{\epsilon}^{\prime}, y-\delta} \in D[a, b]^{2}$.

$N$ th-degree judicious appetite for (aggravated) risk thus means that more of a zero-mean risk in the first attribute (aggravated by a reduction in the second) becomes less desirable if the zero-mean risk worsens in the sense of an $N$ th-degree risk increase. It is clear that this behavioral condition is quite distinct from that of risk aversion. Importantly, scaling up a zero-mean risk $\tilde{\epsilon}$ in the presence of a background risk $\tilde{w}$ may or may not induce an MPIR and hence may or may not be undesirable to a risk averse individual. As shown in what follows, this depends critically on the correlation between $\tilde{\epsilon}$ and $\tilde{w}$.

Wright (1987) puts forward the notion of negative expectation dependence: $\tilde{x}$ is negative expectation dependent on $\tilde{y}$ if $E[\tilde{x} \mid \tilde{y} \leq y] \geq E[\tilde{x}]$ for all $y$. That is, given the truncation $\tilde{y} \leq y$, the expectation of $\tilde{x}$ is revised upward.

Definition $7 \tilde{x}$ is positive (negative) expectation dependent on $\tilde{y}$ if $E[\tilde{x} \mid \tilde{y} \leq y] \leq(\geq) E[\tilde{x}]$ for all $y$.

By a key property of expectation dependence established in Wright (1987), we can formally relate the distributional effect of upscaling a risk in the presence of another to the correlation between the risks.

Lemma 3 Given $\alpha \geq 0$ and risky prospects $\tilde{w}$ and $\tilde{\epsilon}$ with $E[\tilde{\epsilon}]=0$, an increase in $\alpha$ induces an MPIR in $F_{\tilde{w}+\alpha \tilde{\epsilon}}$ if and only if $\tilde{\epsilon}$ is positive expectation dependent on $\tilde{w}$.

This regularity on the distributional effect of upscaling a risk in the presence of another provides for the characterization of risk aversion in our analytical framework assuming only utility-representable 
preferences in the next section as well as the characterization of $N$ th-degree judicious appetite for (aggravated) risk in the EU framework given in the Appendix.

\section{Changes in Risk and Optimal Decisions under Risk with Utility- Representable Preferences}

We set out an analytical framework for decision-making under risk assuming only utility-representable preferences. The analytical framework assumes, as do existing models, that an individual's preference ordering on $D[a, b]^{n}, \succsim^{(n)}$, is complete, transitive and "continuous in distribution" and is thus representable by a continuous real-valued preference functional $V^{(n)}(\cdot)$ on $D[a, b]^{n}$, i.e.,

$$
F_{\tilde{x}_{1}, \ldots, \tilde{x}_{n}} \succsim^{(n)} F_{\tilde{x}_{1}^{\prime}, \ldots, \tilde{x}_{n}^{\prime}} \text { if and only if } V^{(n)}\left(F_{\tilde{x}_{1}, \ldots, \tilde{x}_{n}}\right) \geq V^{(n)}\left(F_{\tilde{x}_{1}^{\prime}, \ldots, \tilde{x}_{n}^{\prime}}\right) \text {. }
$$

The EU model, in particular, is the special case where

$$
V^{(n)}\left(F_{\tilde{x}_{1}, \ldots, \tilde{x}_{n}}\right)=E\left[u\left(\tilde{x}_{1}, \ldots, \tilde{x}_{n}\right)\right]
$$

with $u(\cdot, \ldots, \cdot)$ being the VNM utility function ${ }^{14}$ and EU analysis is essentially the analysis of how conditions on $u(\cdot)$ affect the expected value of the distribution $F_{u\left(\tilde{x}_{1}, \ldots, \tilde{x}_{n}\right)}$. In contrast to the EU and other existing models, however, we make no further assumption to cardinalize $V^{(n)}(\cdot)$ in any way. In particular, there is no assumption that implies any form of event-separability and thus there is no explicit specification of how $V^{(n)}\left(F_{\tilde{x}_{1}, \ldots, \tilde{x}_{n}}\right)$ relates to the utility of each of $\left(\tilde{x}_{1}, \ldots, \tilde{x}_{n}\right)$ 's possible outcomes obtained with certainty. Neither is there any assumption of differentiability on $V^{(n)}(\cdot)$ (which requires a cardinalization) to obtain the "local utility" function. As a result, there is no analogue of the VNM utility function, the simple and elegant characterizations of regularities on risk attitudes in terms of which explain much of the EU model's lasting popularity as remarked earlier. For characterizing behavioral regularities, we use instead an ordinal utility function of "decision parameters" defined as follows: Note first that, with appropriately chosen zero-mean random variables $\left(\tilde{\epsilon}_{j}\right)_{j=1}^{n},\left(\tilde{z}_{j}\right)_{j=1}^{n},\left(\tilde{\epsilon}_{j}^{\prime}\right)_{j=1}^{n}$, and $\left(\tilde{z}_{j}^{\prime}\right)_{j=1}^{n}$, forming

$$
\gamma F_{\mu_{1}+\tilde{z}_{1}+\alpha_{1} \tilde{\epsilon}_{1}, \ldots, \mu_{n}+\tilde{z}_{n}+\alpha_{n} \tilde{\epsilon}_{n}}+(1-\gamma) F_{\mu_{1}^{\prime}+\tilde{z}_{1}^{\prime}+\alpha_{1}^{\prime} \tilde{\epsilon}_{1}^{\prime}, \ldots, \mu_{n}^{\prime}+\tilde{z}_{n}^{\prime}+\alpha_{n}^{\prime} \tilde{\epsilon}_{n}^{\prime}}
$$

where $\gamma \in[0,1],\left(\mu_{j}\right)_{j=1}^{n},\left(\alpha_{j}\right)_{j=1}^{n},\left(\mu_{j}^{\prime}\right)_{j=1}^{n}$, and $\left(\alpha_{j}^{\prime}\right)_{j=1}^{n}$ are real-valued "decision parameters", any

\footnotetext{
${ }^{14}$ Equivalently, it is the special case with the imposition of the "independence axiom" in addition to completeness, transitivity, and continuity in distribution, as is well-known.
} 
changes in probability distribution resulting from a decision under risk can be represented by changes in the decision parameters. Secondly, given the representation of $\succsim^{(n)}$ by $V^{(n)}(\cdot)$,

$$
\begin{aligned}
& U^{(n)}\left(\gamma, \mu_{1}, \alpha_{1}, \ldots, \mu_{n}, \alpha_{n}, \mu_{1}^{\prime}, \alpha_{1}^{\prime}, \ldots, \mu_{n}^{\prime}, \alpha_{n}^{\prime} ; \tilde{\epsilon}_{1}, \tilde{z}_{1}, \ldots, \tilde{\epsilon}_{n}, \tilde{z}_{n}, \tilde{\epsilon}_{1}^{\prime}, \tilde{z}_{1}^{\prime}, \ldots, \tilde{\epsilon}_{n}^{\prime}, \tilde{z}_{n}^{\prime}\right) \\
& \quad \equiv V^{(n)}\left(\gamma F_{\mu_{1}+\tilde{z}_{1}+\alpha_{1} \tilde{\epsilon}_{1}, \ldots, \mu_{n}+\tilde{z}_{n}+\alpha_{n} \tilde{\epsilon}_{n}}+(1-\gamma) F_{\left.\mu_{1}^{\prime}+\tilde{z}_{1}^{\prime}+\alpha_{1}^{\prime} \tilde{\epsilon}_{1}^{\prime}, \ldots, \mu_{n}^{\prime}+\tilde{z}_{n}^{\prime}+\alpha_{n}^{\prime} \tilde{\epsilon}_{n}^{\prime}\right)}\right.
\end{aligned}
$$

is well-defined for values of the decision parameters such that

$$
\gamma F_{\mu_{1}+\tilde{z}_{1}+\alpha_{1} \tilde{\epsilon}_{1}, \ldots, \mu_{n}+\tilde{z}_{n}+\alpha_{n} \tilde{\epsilon}_{n}}+(1-\gamma) F_{\mu_{1}^{\prime}+\tilde{z}_{1}^{\prime}+\alpha_{1}^{\prime} \tilde{\epsilon}_{1}^{\prime}, \ldots, \mu_{n}^{\prime}+\tilde{z}_{n}^{\prime}+\alpha_{n}^{\prime} \tilde{\epsilon}_{n}^{\prime}} \in D[a, b]^{n}
$$

without any additional assumption on the preference relation $\succsim^{(n)}$. Any decision-relevant behavioral regularities can thus in principle be characterized by the properties of the function. ${ }^{15}$ This analytical framework can model all the problems that the EU framework can model since any decision problem that can be formulated in terms of the decision's effect on the expected value of the distribution $F_{u\left(\tilde{x}_{1}, \ldots, \tilde{x}_{n}\right)}$ can clearly be formulated in terms of its effect on the distribution $F_{\tilde{x}_{1}, \ldots, \tilde{x}_{n}}$ itself.

Rothschild and Stiglitz (1971) consider in the EU framework the class of problems of the form

$$
\max _{q} E u(\tilde{x}, q)
$$

and obtain conditions on the VNM utility function sufficient for comparative statics results. Machina (1989) subsequently shows that the characterizations in terms of the VNM utility function derived for comparative statics results can be generalized to those in terms of the "local utility function" provided that the preference functional is Frechet differentiable. This class of problems can be seen to be a sub-class of problems of the following form

$$
\max _{q} V\left(F_{\tilde{w}+q \tilde{x}, \Gamma-\beta q}\right)
$$

If we let $q$ denote labor supply, $\tilde{x}$ the (potentially uncertain) wage rate, $\tilde{w}$ the (potentially uncertain) non-wage income, $\Gamma$ the total time endowment and $\beta=1,(2)$ becomes the standard labor supply problem. Likewise, letting $q$ be the amount of saving, $\tilde{w}$ the second-period uncertain income, $\tilde{x}$ the gross return for saving, $\Gamma$ the first-period income and $\beta=1$, (2) becomes the two-period consumption/saving problem. If we let $\beta=0$, on the other hand, (2) is just the class of problems

\footnotetext{
${ }^{15}$ Among the problems of choice under risk that the EU framework can model are those where an individual effectively chooses the likelihoods of a lottery's different possible outcomes. These problems can be modeled in our framework as an individual choosing a probability mixture $\gamma \in[0,1]$ of two distributions $F_{\tilde{x}}$ and $F_{\tilde{x}^{\prime}}: \lambda F_{\tilde{x}}+(1-\lambda) F_{\tilde{x}^{\prime}}$. And the special case of (1) where $n=1$ :

$$
U^{i(1)}\left(\gamma, \mu_{1}, \mu_{1}^{\prime} ; \tilde{z}_{1}, \tilde{z}_{1}^{\prime}\right) \equiv V^{i(1)}\left(\gamma F_{\mu_{1}+\tilde{z}_{1}+\alpha_{1} \tilde{\epsilon}_{1}}+(1-\gamma) F_{\mu_{1}^{\prime}+\tilde{z}_{1}^{\prime}+\alpha_{1}^{\prime} \tilde{\epsilon}_{1}^{\prime}}\right)
$$

can be used to characterize relevant behavioral regularities.
} 
with the payoff taking the form $\tilde{w}+q \tilde{x}$, prominent examples of which include the following. First, if $\tilde{w}_{0}$ denotes the random initial wealth and $q$ the amount invested in a risky asset whose random return rate is $\tilde{r}$ and there is a riskless asset whose return rate is $r_{0}$, then the final wealth in this portfolio-choice problem is $\tilde{y}=\left(1+r_{0}\right)\left(\tilde{w}_{0}-q\right)+q(1+\tilde{r})=\left(1+r_{0}\right) \tilde{w}_{0}+q\left(\tilde{r}-r_{0}\right) \equiv \tilde{w}+q \tilde{x}$, where $\tilde{w} \equiv\left(1+r_{0}\right) \tilde{w}_{0}$ and $\tilde{x} \equiv \tilde{r}-r_{0}$. A variant of the problem takes the following form: An invester has to allocate his potentially random initial wealth $\tilde{w}_{0}$ between two risky assets, which are identified with their return rates $\tilde{r}$ and $\tilde{r}_{0}$. Letting $q$ be the amount invested in asset $\tilde{r}$, his final wealth is then $\tilde{y}=\left(1+\tilde{r}_{0}\right)\left(\tilde{w}_{0}-q\right)+q(1+\tilde{r})=\left(1+\tilde{r}_{0}\right) \tilde{w}_{0}+q\left(\tilde{r}-\tilde{r}_{0}\right) \equiv \tilde{w}+q \tilde{x}$, where $\tilde{w} \equiv\left(1+\tilde{r}_{0}\right) \tilde{w}_{0}$ and $\tilde{x} \equiv \tilde{r}-\tilde{r}_{0}$. Secondly, letting $\tilde{l}$ be the random insurable loss, $c$ the coinsurance rate, $\pi=c\left(\mu_{\tilde{l}}+\delta\right)$ the insurance premium, and $\tilde{w}_{0}$ still the random initial wealth, the final wealth in this insurancepurchasing problem is given by $\tilde{y}=\tilde{w}_{0}-\tilde{l}+c \tilde{l}-c\left(\mu_{\tilde{l}}+\delta\right)=\left[\tilde{w}_{0}-\mu_{\tilde{l}}-\delta\right]+(1-c)\left(\mu_{\tilde{l}}-\tilde{l}+\delta\right) \equiv \tilde{w}+q \tilde{x}$ where $\tilde{w} \equiv\left[\tilde{w}_{0}-\mu_{\tilde{l}}-\delta\right], q \equiv(1-c)$, and $\tilde{x} \equiv\left(\mu_{\tilde{l}}-\tilde{l}+\delta\right)$.

For analyzing this class of problems, the special case of (1) where $\gamma=1$ and $n=2$ can be used:

$$
U\left(\mu_{1}, \alpha_{1}, \mu_{2}, \alpha_{2} ; \tilde{\epsilon}_{1}, \tilde{z}_{1}, \tilde{\epsilon}_{2}, \tilde{z}_{2}\right) \equiv V\left(F_{\mu_{1}+\tilde{z}_{1}+\alpha_{1} \tilde{\epsilon}_{1}, \mu_{2}+\tilde{z}_{2}+\alpha_{2} \tilde{\epsilon}_{2}}\right)
$$

which we further simplify by assuming $\alpha_{1}=\alpha, \alpha_{2}=0, \tilde{z}_{1}=\tilde{z}, \tilde{\epsilon}_{1}=\tilde{\epsilon}, \tilde{z}_{2}=\tilde{\epsilon}_{2}=0$ to arrive at

$$
U\left(\mu_{1}, \alpha, \mu_{2} ; \tilde{\epsilon}, \tilde{z}\right) \equiv V\left(F_{\mu_{1}+\tilde{z}+\alpha \tilde{\epsilon}, \mu_{2}}\right) .
$$

Setting $\mu_{1}=E[\tilde{w}]+q E[\tilde{x}], \alpha=q, \mu_{2}=\Gamma-\beta q, \tilde{\epsilon}=\tilde{x}-E[\tilde{x}]$ and $\tilde{z}=\tilde{w}-E[\tilde{w}]$, we get

$$
U(E[\tilde{w}]+q E[\tilde{x}], q, \Gamma-\beta q ; \tilde{w}-E[\tilde{w}], \tilde{x}-E[\tilde{x}])=V\left(F_{\tilde{w}+q \tilde{x}, \Gamma-\beta q}\right) .
$$

We first characterize mean-monotonicity and risk aversion in terms of the properties of the function $U\left(\mu_{1}, \alpha, \mu_{2} ; \tilde{\epsilon}, \tilde{z}\right)$ as follows.

\section{Proposition 1}

(i) $U\left(\mu_{1}, \alpha, \mu_{2} ; \tilde{\epsilon}, \tilde{z}\right)$ is increasing in $\mu_{1}$ and $\mu_{2}$ for all $\left(\mu_{1}, \alpha, \mu_{2}\right) \in R^{3}$ and $\tilde{\epsilon}$ and $\tilde{z}$ such that $F_{\mu_{1}+\tilde{z}+\alpha \tilde{\epsilon}, \mu_{2}} \in D[a, b]^{2}$ and $E[\tilde{\epsilon}]=E[\tilde{z}]=0$ if and only if $\succsim$ exhibits mean-monotonicity.

(ii) $U\left(\mu_{1}, \alpha, \mu_{2} ; \tilde{\epsilon}, \tilde{z}\right)$ is decreasing in $\alpha$ for all $\left(\mu_{1}, \alpha, \mu_{2}\right) \in R \times R_{+} \times R$ and $\tilde{\epsilon}$ and $\tilde{z}$ such that $F_{\mu_{1}+\tilde{z}+\alpha \tilde{\epsilon}, \mu_{2}} \in D[a, b]^{2}, E[\tilde{\epsilon}]=E[\tilde{z}]=0$ and $\tilde{\epsilon}$ is positive expectation dependent on $\tilde{z}$ if and only if $\succsim$ exhibits risk aversion. 
Mean-monotonicity can thus be completely characterized by the utility function $U\left(\mu_{1}, \alpha, \mu_{2} ; \tilde{\epsilon}, \tilde{z}\right)$ being increasing in $\mu_{1}$ and $\mu_{2}$ while assuming $\tilde{\epsilon}$ is positive expectation dependent on $\tilde{z}$, risk aversion is completely characterized by the utility function being decreasing in $\alpha$.

Given a value for $\bar{u}, \mu_{2}$, and $\theta, U^{i}\left(\mu_{1}, \alpha, \mu_{2}-\theta \alpha ; \tilde{\epsilon}, \tilde{z}\right)=\bar{u}$ defines a $\mu_{1}-\alpha$ indifference curve. We allow for the possibility of the $\mu_{1}-\alpha$ indifference curves not being smooth everywhere and are agnostic about where points of non-smoothness (i.e., kinks) occur. ${ }^{16}$ Define $\Delta \mu\left(\mu_{1}, \alpha, \mu_{2}, \delta, \theta ; \tilde{\epsilon}, \tilde{z}\right)$ by the solution for $\Delta \mu$ to

$$
U\left(\mu_{1}+\Delta \mu, \alpha+\delta, \mu_{2}-\theta(\alpha+\delta) ; \tilde{\epsilon}, \tilde{z}\right)=U\left(\mu_{1}, \alpha, \mu_{2}-\theta \alpha ; \tilde{\epsilon}, \tilde{z}\right)
$$

and the $\mu_{1}-\alpha$ marginal rates of substitution as

$$
S_{+}\left(\mu_{1}, \alpha, \mu_{2}, \theta ; \tilde{\epsilon}, \tilde{z}\right) \equiv \lim _{\delta \rightarrow 0^{+}} \frac{\Delta \mu\left(\mu_{1}, \alpha, \mu_{2}, \delta, \theta ; \tilde{\epsilon}, \tilde{z}\right)}{\delta}
$$

and

$$
S_{-}\left(\mu_{1}, \alpha, \mu_{2}, \theta ; \tilde{\epsilon}, \tilde{z}\right) \equiv \lim _{\delta \rightarrow 0^{-}} \frac{\Delta \mu\left(\mu_{1}, \alpha, \mu_{2}, \delta, \theta ; \tilde{\epsilon}, \tilde{z}\right)}{\delta}
$$

Prudence and $N$ th-degree judicious appetite for (aggravated) risk can be completely characterized in terms of these marginal rates of substitution.

\section{Proposition 2}

(i) $\succsim$ exhibits $N$ th-degree judicious appetite for aggravated risk if and only if

$$
S_{+}\left(\mu_{1}, \alpha, \mu_{2}, \theta ; \tilde{\epsilon}^{\prime}, \tilde{z}\right) \geq S_{+}\left(\mu_{1}, \alpha, \mu_{2}, \theta ; \tilde{\epsilon}, \tilde{z}\right) \text { and } S_{-}\left(\mu_{1}, \alpha, \mu_{2}, \theta ; \tilde{\epsilon}^{\prime}, \tilde{z}\right) \geq S_{-}\left(\mu_{1}, \alpha, \mu_{2}, \theta ; \tilde{\epsilon}, \tilde{z}\right)
$$

for all $\left(\mu_{1}, \alpha, \mu_{2}, \theta\right) \in\left(R \times R_{+}\right)^{2}$ and $\tilde{\epsilon}, \tilde{z}$, and $\tilde{\epsilon}^{\prime}$ such that $F_{\mu+\tilde{z}+\alpha \tilde{\epsilon}, \mu_{2}} \in D[a, b]^{2}, F_{\mu+\tilde{z}+\alpha \tilde{\epsilon}^{\prime}, \mu_{2}} \in$ $D[a, b]^{2}, E[\tilde{\epsilon}]=E[\tilde{z}]=0$ and $F_{\tilde{\epsilon}^{\prime} \mid \tilde{z}}$ is an $N$ th-degree risk increase of $F_{\tilde{\epsilon} \mid \tilde{z}}$.

(ii) $\succsim$ exhibits $N$ th-degree judicious appetite for risk if and only if

$$
S_{+}\left(\mu_{1}, \alpha, \mu_{2}, 0 ; \tilde{\epsilon}^{\prime}, \tilde{z}\right) \geq S_{+}\left(\mu_{1}, \alpha, \mu_{2}, 0 ; \tilde{\epsilon}, \tilde{z}\right) \text { and } S_{-}\left(\mu_{1}, \alpha, \mu_{2}, 0 ; \tilde{\epsilon}^{\prime}, \tilde{z}\right) \geq S_{-}\left(\mu_{1}, \alpha, \mu_{2}, 0 ; \tilde{\epsilon}, \tilde{z}\right)
$$

for all $\left(\mu_{1}, \alpha, \mu_{2}\right) \in R \times R_{+} \times R$ and $\tilde{\epsilon}, \tilde{z}$, and $\tilde{\epsilon}^{\prime}$ such that $F_{\mu+\tilde{z}+\alpha \tilde{\epsilon}, \mu_{2}} \in D[a, b]^{2}, F_{\mu+\tilde{z}+\alpha \tilde{\epsilon}^{\prime}, \mu_{2}} \in$ $D[a, b]^{2}, E[\tilde{\epsilon}]=E[\tilde{z}]=0$ and $F_{\tilde{\epsilon}^{\prime} \mid \tilde{z}}$ is an $N$ th-degree risk increase of $F_{\tilde{\epsilon} \mid \tilde{z}}$.

\footnotetext{
${ }^{16}$ It can be easily shown that the $\mu_{1}-\alpha$ indifference curves will be smooth everywhere if we assume, as does Machina (1982a), that $V(F)$ is " $L_{1}$-Frechet differentiable" (for a formal definition of which, see, for example, Wang (1993)). As a general behavioral (and hence ordinal) condition, however, smooth $\mu_{1}-\alpha$ indifference curves do not require cardinal properties such as the differentiability of the preference functional. An analogy can be made with standard consumer theory where smooth indifference curves are normally assumed but not a cardinal utility function.
} 
(iii) $\succsim$ exhibits $N$ th-degree prudence if and only if

$$
\begin{aligned}
& S_{+}\left(\mu_{1}, \alpha, \mu_{2}, 1 ; 0, \tilde{z}\right) \geq S_{+}\left(\mu_{1}, \alpha, \mu_{2}, 1 ; 0, \tilde{z}^{\prime}\right) \text { and } S_{-}\left(\mu_{1}, \alpha, \mu_{2}, 1 ; 0, \tilde{z}\right) \geq S_{-}\left(\mu_{1}, \alpha, \mu_{2}, 1 ; 0, \tilde{z}^{\prime}\right) \\
& \text { for all }\left(\mu_{1}, \alpha, \mu_{2}\right) \in R^{3} \text { and } \tilde{z} \text { and } \tilde{z}^{\prime} \text { such that } F_{\mu+\tilde{z}, \mu_{2}} \in D[a, b]^{2}, F_{\mu+\tilde{z}^{\prime}, \mu_{2}} \in D[a, b]^{2}, E[\tilde{z}]=0 \\
& \text { and } F_{\tilde{z}^{\prime}} \text { is an } N \text { th-degree risk increase of } F_{\tilde{z}} \text {. }
\end{aligned}
$$

That is, $N$ th-degree judicious appetite for (aggravated) risk is equivalent to the $\mu_{1}-\alpha$ marginal rates of substitution for $\theta \geq 0(\theta=0)$ being raised by an $N$ th-degree risk increase in the "endogenous risk" $\tilde{\epsilon}$ while $N$ th-degree prudence is equivalent to the $\mu_{1}-\alpha$ marginal rates of substitution for $\theta=1$ and $\tilde{\epsilon}=0$ being reduced by an $N$ th-degree risk increase in the "background risk" $\tilde{z}$. These characterizations are instrumental in establishing our main result that relates formally these attitudes towards changes in risk to optimal decisions under risk.

Proposition 3 Suppose $\succsim$ exhibits mean-monotonicity and $C$-diversification and $q^{*}(\tilde{x}, \tilde{w}, \Gamma, \beta) \equiv$ $\arg \max _{q} V\left(F_{\tilde{w}+q \tilde{x}, \Gamma-\beta q}\right)$ is non-negative. Then

(i) $q^{*}\left(\tilde{x}^{\prime}, \tilde{w}, \Gamma, \beta\right) \leq q^{*}(\tilde{x}, \tilde{w}, \Gamma, \beta)$ for $(\Gamma, \beta) \in R \times R_{+}$and $F_{\tilde{x}^{\prime} \mid \tilde{w}}$ being an $N$ th-degree risk increase of $F_{\tilde{x} \mid \tilde{w}}$ if and only if $\succsim$ exhibits $N$ th-degree judicious appetite for aggravated risk.

(ii) $q^{*}\left(\tilde{x}^{\prime}, \tilde{w}, \Gamma, 0\right) \leq q^{*}(\tilde{x}, \tilde{w}, \Gamma, 0)$ for $\Gamma \in R$ and $F_{\tilde{x}^{\prime} \mid \tilde{w}}$ being an $N$ th-degree risk increase of $F_{\tilde{x} \mid \tilde{w}}$ if and only if $\succsim$ exhibits $N$ th-degree judicious appetite for risk.

(iii) $q^{*}\left(\mu_{\tilde{x}}, \tilde{w}^{\prime}, \Gamma, 1\right) \geq q^{*}\left(\mu_{\tilde{x}}, \tilde{w}, \Gamma, 1\right)$ for all $\Gamma \in R$ and $F_{\tilde{w}^{\prime}}$ being an $N$ th-degree risk increase over $F_{\tilde{w}}$ if and only if $\succsim$ exhibits $N$ th-degree prudence.

Under C-diversification, since the objective function $V\left(F_{\tilde{w}+q \tilde{x}, \Gamma-\beta q}\right)$ is quasi-concave in $q$, the solution to the optimization problem can be characterized by the first-order conditions. The condition, together with mean-monotonicity (i.e., an upward shift of a bivariate distribution in the direction of either variable is preferred), thus enables the regularities on the marginal rates of substitution established in Proposition 2 to imply regularities on optimal decisions. ${ }^{17}$

In the labor supply or precautionary saving setting where $\beta=1$, Proposition 3(i) shows that, assuming $N$ th-degree judicious appetite for aggravated risk, labor supply or saving decreases if the wage rate or the gross saving return undergoes an $N$ th-degree risk increase. Given the equivalence

\footnotetext{
${ }^{17}$ The assumption of the preference conditions mean-monotonicity and C-diversification can be seen to correspond to the usual assumption in the EU framework that the VNM utility function is increasing and concave in its attributes since, as explained earlier, in the EU framework, an increasing and concave VNM utility function is necessary and sufficient for these preference conditions.
} 
of choosing $q$ to maximize $U(E[\tilde{w}]+q E[\tilde{x}], q, \Gamma-q ; \tilde{\epsilon}, \tilde{z})$ to choosing $\mu_{1}$ and $q$ to maximize $U\left(\mu_{1}, q, \Gamma-\right.$ $q ; \tilde{\epsilon}, \tilde{z}$ ) subject to the constraint $\mu_{1}=E[\tilde{w}]+q E[\tilde{x}]$, the effect of the risk increase from $\tilde{x}$ to $\tilde{x}^{\prime}$ (in the particular case where the $\mu_{1}-\alpha$ indifference curves are smooth) can be illustrated graphically as follows. ${ }^{18}$
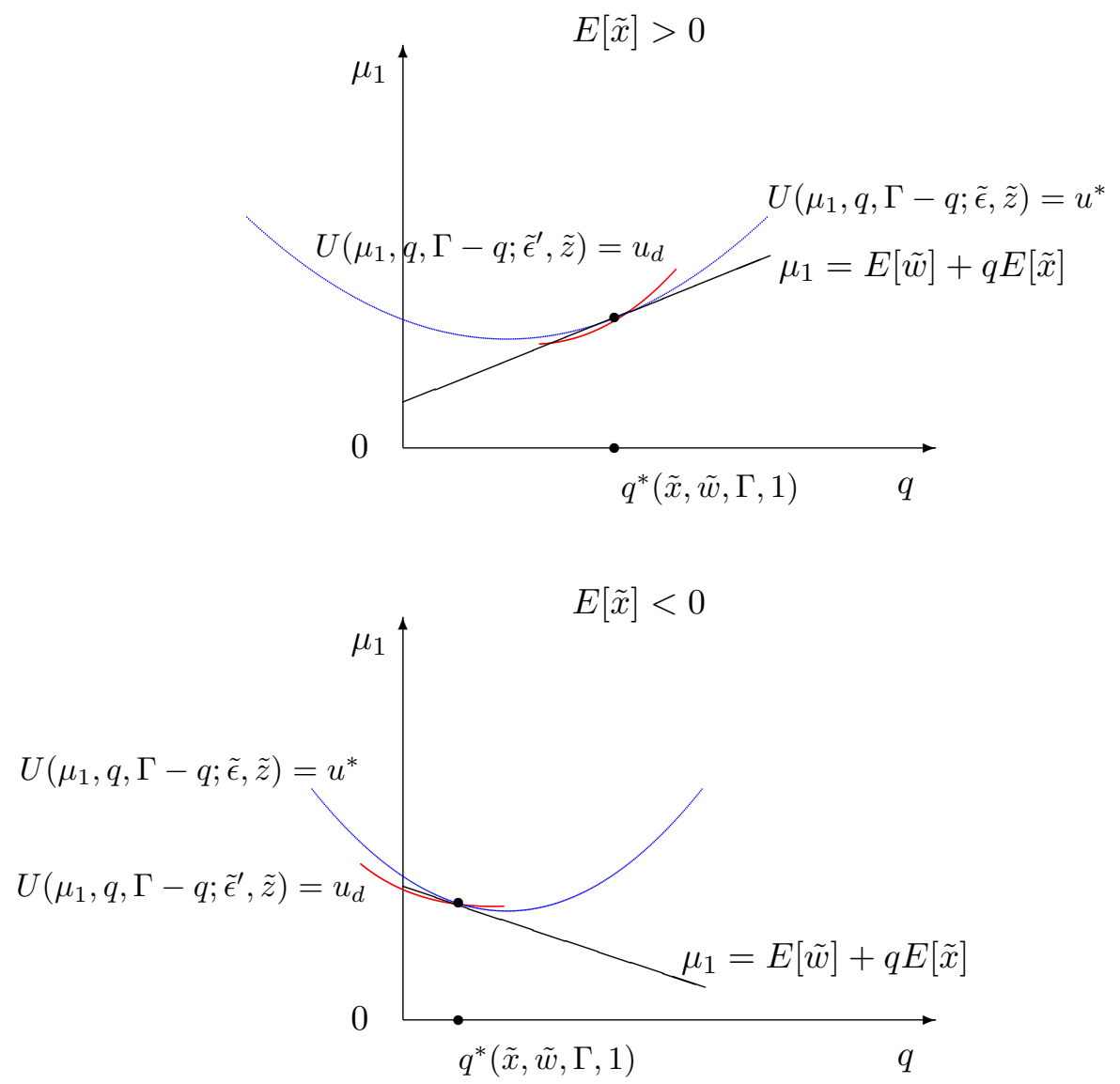

The risk increase from $\tilde{x}$ to $\tilde{x}^{\prime}$ leads to a lower optimal $q$, more intuitively, since it makes an increase in $q$ less desirable whether an increase in $q$ from $q^{*}(\tilde{x}, \tilde{w}, \Gamma, 1)$ is desirable as in the case of $E[\tilde{x}]<0$ (where the indifference curve is downward-sloping) or not as in the case of $E[\tilde{x}]>0$ (where the indifference curve is upward-sloping), which holds if $\succsim$ exhibits $N$ th-degree judicious appetite for aggravated risk (Proposition 2 (i)).

Proposition 3(ii) pertains to the case where $\beta=0$ and $\tilde{x}$ undergoes a risk increase while $\tilde{w}$ remains unchanged. As mentioned previously, this is the class of problems with the payoff in the

\footnotetext{
${ }^{18}$ Under C-diversification, a $\mu_{1}-q$ indifference curve defined by $U\left(\mu_{1}, q, \Gamma-q ; \tilde{\epsilon}, \tilde{z}\right)=\bar{u}$ is convex. The precise shape and position of a $\mu_{1}-q$ indifference curve depend however on the relationship between $\tilde{\epsilon}$ and $\tilde{z}$. It can be shown that if $\tilde{\epsilon}$ is negative expectation dependent on $\tilde{z}$, there can be an $\hat{q}>0$ such that the curve is upward-sloping for $q>\hat{q}$ and downward-sloping for $q<\hat{q}$.
} 
form $\tilde{w}+q \tilde{x}$ encompassing the two-asset portfolio choice problem and the insurance-purchasing problem as prominent examples. For concreteness and ease of comparison with existing results, consider the case where an investor has to allocate his wealth $\tilde{w}_{0}$ between two risky assets identified with their return rates $\tilde{r}$ and $\tilde{r}_{0}$ where $\tilde{w}_{0}$ is independent of $\tilde{r}$ and $\tilde{r}_{0}$. The result shows that $N$ thdegree judicious appetite for risk is necessary and sufficient for an $N$ th-degree risk increase in $F_{\tilde{r} \mid \tilde{w}_{0}\left(1+\tilde{r}_{0}\right)}$ to imply a lower investment in the asset $\tilde{r}$. As well as identifying the necessary and sufficient condition on the preferences over distributions for the comparative statics result, this can be seen as an extension of previous results obtained in the EU framework by Rothschild and Stiglitz (1971), Cheng, Magill, and Shafer (1987), Hadar and Seo (1990), and Chiu, Eeckhout, and Rey (2012) to all utility-representable preferences.

Lastly, Proposition 3 (iii) is concerned with the case where $\tilde{x}$ is degenerate, i.e., $\tilde{x} \equiv E[\tilde{x}]$, and $\beta=1$. In the labor supply or precautionary saving setting (where $q$ denotes labor supply or the amount of saving, $\tilde{x}$ the wage rate or the gross return on saving, $\tilde{w}$ the uncertain non-wage income or uncertain second-period income, and $\Gamma$ the total time endowment or the first-period income), Proposition 3 (iii) shows that labor supply or precautionary saving rises in response to an $N$ th-degree risk increase in the non-wage income or second-period income if and only if $\succsim$ exhibits Nth-degree prudence.

\section{Conclusion}

This paper sets out a general analytical framework for decision-making under risk assuming only utility-representable preferences. Focusing on the effects of changes in risk on optimal decisions under risk, we show that a version of the general framework can be used to establish in the class of problems considered by Rothschild and Stiglitz (1971) and Machina (1989) the equivalence between behavioral assumptions and the characteristics of the preference-representing utility function, which are in turn necessary and sufficient for comparative statics results. More specifically, we show in the setting of the two-period consumption-saving problem or the standard labor supply problem that a preference condition, which we term " $N$ th-degree prudence", is necessary and sufficient for precautionary saving or labor supply to rise as a result of the second-period income or non-wage income undergoing an $N$ th-degree risk increase while one, which we term " $N$ th-degree judicious appetite for aggravated risk", is sufficient for a $N$ th-degree risk increase in the second-period interest rate or 
wage rate to imply lower precautionary saving or labor supply. Furthermore in the portfolio choice setting with one risky asset and one riskless asset the weaker condition of " $N$ th-degree judicious appetite for risk" is shown to be necessary and sufficient for an $N$ th-degree risk increase in the risky asset return to imply a lower investment in the asset.

\section{Appendix}

\section{A1. Proofs.}

The proof of Lemma 3 uses the following result established in Wright (1987).

Lemma 4 (Wright (1987)) $\tilde{x}$ is positive (negative) expectation dependent on $\tilde{y}$ if and only if $\operatorname{cov}[\tilde{x}, \psi(\tilde{y})] \geq(\leq) 0$ for all increasing function $\psi$ for which the covariance exists.

Proof of Lemma 3. Let $\phi(\alpha) \equiv E u(\tilde{w}+\alpha \tilde{\epsilon})$. Then $\phi^{\prime}(\alpha)=E \tilde{\epsilon} u^{\prime}(\tilde{w}+\alpha \tilde{\epsilon})=\operatorname{cov}\left[\tilde{\epsilon}, u^{\prime}(\tilde{w}+\alpha \tilde{\epsilon})\right]$. If $\tilde{\epsilon}$ is positive expectation dependent on $\tilde{w}$, then by Lemma $4 \phi^{\prime}(0) \leq 0$ for all concave function $u$, which, coupled with $\phi^{\prime \prime}(\alpha)=E \tilde{\epsilon}^{2} u^{\prime \prime}(\tilde{w}+\alpha \tilde{\epsilon}) \leq 0$ for all concave $u$, implies that, for all concave $u$, $\phi^{\prime}(\alpha) \leq 0$ for $\alpha \geq 0$. Lemma 1 thus implies that an increase in $\alpha$ induces in $F_{\tilde{w}+\alpha \tilde{\epsilon}}$ an MPIR for $\alpha \geq 0$. Conversely, if $\tilde{\epsilon}$ is not positive expectation dependent on $\tilde{w}$, by Lemma 4 it is possible to have $\phi^{\prime}(0)>0$ for a concave function $u$. Then by Lemma 1 an increase in $\alpha$ clearly cannot induce in $F_{\tilde{w}+\alpha \tilde{\epsilon}}$ an MPIR for all $\alpha \geq 0$.

\section{Proof of Proposition 1.}

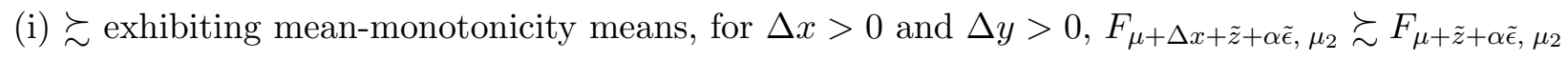
and $F_{\mu+\tilde{z}+\alpha \tilde{\epsilon}, \mu_{2}+\Delta y} \succsim F_{\mu+\tilde{z}+\alpha \tilde{\epsilon}, \mu_{2}}$, which is clearly equivalent to $U\left(\mu_{1}, \alpha, \mu_{2} ; \tilde{\epsilon}, \tilde{z}\right)$ being increasing in $\mu_{1}$ and $\mu_{2}$.

(ii) In view of Lemma $3, \succsim$ exhibiting risk aversion if and only if for $\alpha \geq 0$ and $\Delta \alpha>0$, $F_{\mu+\tilde{z}+\alpha \tilde{\epsilon}, \mu_{2}} \succsim F_{\mu+\tilde{z}+(\alpha+\Delta \alpha) \tilde{\epsilon}, \mu_{2}}$, which is clearly equivalent to $U\left(\mu_{1}, \alpha, \mu_{2} ; \tilde{\epsilon}, \tilde{z}\right)$ being decreasing in $\alpha$ for $\alpha \geq 0$.

\section{Proof of Proposition 2.}

(i) $\succsim$ exhibits $N$ th-degree judicious appetite for aggravated risk if and only if $F_{\mu+\tilde{z}+\alpha \tilde{\epsilon}, \mu_{2}} \sim$ $F_{\mu+\Delta \mu+\tilde{z}+(\alpha+\delta) \tilde{\epsilon}, \mu_{2}-\theta(\alpha+\delta)}$ implies $F_{\mu+\tilde{z}+\alpha \tilde{\epsilon}^{\prime}, \mu_{2}} \succsim(\precsim) F_{\mu+\Delta \mu+\tilde{z}+(\alpha+\delta) \tilde{\epsilon}^{\prime}, \mu_{2}-\theta(\alpha+\delta)}$ for $\delta>(<) 0$, $\theta \geq 0, \alpha \geq 0$ and $F_{\tilde{\epsilon}^{\prime} \mid \tilde{z}}$ being an $N$ th-degree risk increase of $F_{\tilde{\epsilon} \mid \tilde{z}}$. Equivalently, 


$$
U\left(\mu+\Delta \mu\left(\mu_{1}, \alpha, \mu_{2}, \delta, \theta ; \tilde{\epsilon}, \tilde{z}\right), \alpha+\delta, \mu_{2}-\theta(\alpha+\delta) ; \tilde{\epsilon}, \tilde{z}\right)=U\left(\mu_{1}, \alpha, \mu_{2} ; \tilde{\epsilon}, \tilde{z}\right)
$$

implies

$$
U\left(\mu+\Delta \mu\left(\mu_{1}, \alpha, \mu_{2}, \delta, \theta ; \tilde{\epsilon}, \tilde{z}\right), \alpha+\delta, \mu_{2}-\theta(\alpha+\delta) ; \tilde{\epsilon}^{\prime}, \tilde{z}\right) \geq(\leq) U\left(\mu_{1}, \alpha, \mu_{2} ; \tilde{\epsilon}^{\prime}, \tilde{z}\right) .
$$

But given $U\left(\mu_{1}, \alpha, \mu_{2} ; \tilde{\epsilon}^{\prime}, \tilde{z}\right)=U\left(\mu_{1}+\Delta \mu\left(\mu_{1}, \alpha, \mu_{2}, \delta, \theta ; \tilde{\epsilon}^{\prime}, \tilde{z}\right), \alpha+\delta, \mu_{2}-\theta(\alpha+\delta) ; \tilde{\epsilon}^{\prime}, \tilde{z}\right)$, this is equivalent to $\Delta \mu\left(\mu_{1}, \alpha, \mu_{2}, \delta, \theta ; \tilde{\epsilon}, \tilde{z}\right) \leq(\geq) \Delta \mu\left(\mu_{1}, \alpha, \mu_{2}, \delta, \theta ; \tilde{\epsilon}^{\prime}, \tilde{z}\right)$ for $\delta>(<) 0$ and $\theta \geq 0$, which in turn implies and is implied by, for $\left(\mu_{1}, \alpha, \mu_{2}, \theta\right) \in\left(R \times R_{+}\right)^{2}$,

$$
S_{+}\left(\mu_{1}, \alpha, \mu_{2}, \theta ; \tilde{\epsilon}^{\prime}, \tilde{z}\right) \geq S_{+}\left(\mu_{1}, \alpha, \mu_{2}, \theta ; \tilde{\epsilon}, \tilde{z}\right) \text { and } S_{-}\left(\mu_{1}, \alpha, \mu_{2}, \theta ; \tilde{\epsilon}^{\prime}, \tilde{z}\right) \geq S_{-}\left(\mu_{1}, \alpha, \mu_{2}, \theta ; \tilde{\epsilon}, \tilde{z}\right) .
$$

(ii) Analogous to (i).

(iii) $\succsim$ exhibits $N$ th-degree prudence if and only if $F_{\mu_{1}+\tilde{z}, \mu_{2}-\alpha} \sim F_{\mu_{1}+\Delta \mu+\tilde{z}, \mu_{2}-(\alpha+\delta)}$ for $\delta>(<) 0$ implies $F_{\mu_{1}+\tilde{z}^{\prime}, \mu_{2}-\alpha} \precsim(\succsim) F_{\mu_{1}+\Delta \mu+\tilde{z}^{\prime}, \mu_{2}-(\alpha+\delta)}$ for $F_{\tilde{z}^{\prime}}$ being an $N$ th-degree risk increase of $F_{\tilde{z}}$. Equivalently,

$$
U\left(\mu_{1}+\Delta \mu\left(\mu_{1}, \alpha, \mu_{2}, \delta, 1 ; 0, \tilde{z}\right), \alpha+\delta, \mu-\alpha-\delta ; 0, \tilde{z}\right)=U\left(\mu_{1}, \alpha, \mu_{2} ; 0, \tilde{z}\right)
$$

implies

$$
U\left(\mu_{1}+\Delta \mu\left(\mu_{1}, \alpha, \mu_{2}, \delta, 1 ; 0, \tilde{z}\right), \alpha+\delta, \mu-\alpha-\delta ; 0, \tilde{z}^{\prime}\right) \geq(\leq) U\left(\mu_{1}, \alpha, \mu_{2} ; 0, \tilde{z}^{\prime}\right) .
$$

But given $U\left(\mu_{1}, \alpha, \mu_{2} ; 0, \tilde{z}^{\prime}\right)=U\left(\mu_{1}+\Delta \mu\left(\mu_{1}, \alpha, \mu_{2}, \delta, 1 ; 0, \tilde{z}^{\prime}\right), \alpha+\delta, \mu-\alpha-\delta ; 0, \tilde{z}^{\prime}\right)$, this is equivalent to $\Delta \mu\left(\mu_{1}, \alpha, \mu_{2}, \delta, 1 ; 0, \tilde{z}\right) \geq(\leq) \Delta \mu\left(\mu_{1}, \alpha, \mu_{2}, \delta, 1 ; 0, \tilde{z}^{\prime}\right)$ for $\delta>(<) 0$, which in turn implies and is implied by $S_{+}\left(\mu_{1}, \alpha, \mu_{2}, 1 ; 0, \tilde{z}\right) \geq S_{+}\left(\mu_{1}, \alpha, \mu_{2}, 1 ; 0, \tilde{z}^{\prime}\right)$ and $S_{-}\left(\mu_{1}, \alpha, \mu_{2}, 1 ; 0, \tilde{z}\right) \geq$ $S_{-}\left(\mu_{1}, \alpha, \mu_{2}, 1 ; 0, \tilde{z}^{\prime}\right)$ for all $\left(\mu_{1}, \alpha, \mu_{2}\right) \in R \times R_{+} \times R$.

Proof of Proposition 3. This is implied by Proposition 2 and the following result.

Lemma 5 Suppose $\succsim$ exhibits mean-monotonicity and C-diversification, $\tilde{\epsilon}, \tilde{z}, \tilde{\epsilon}^{\prime}$ and $\tilde{z}^{\prime}$ are random variables, $E[\tilde{\epsilon}]=E[\tilde{z}]=0$ and $q^{*}(\tilde{x}, \tilde{w}, \Gamma, \beta) \equiv \arg \max _{q} V\left(F_{\tilde{w}+q \tilde{x}, \Gamma-\beta q}\right)$ is non-negative. Then,

$$
q^{*}\left(\mu_{x}+\tilde{\epsilon}^{\prime}, \mu_{w}+\tilde{z}^{\prime}, \Gamma, \beta\right) \leq(\geq) q^{*}\left(\mu_{x}+\tilde{\epsilon}, \mu_{w}+\tilde{z}, \Gamma, \beta\right)
$$

for all $\left(\mu_{x}, \mu_{w}, \Gamma, \beta\right) \in R^{3} \times R_{+}$if and only if

$$
S_{+}\left(\mu_{1}, \alpha, \mu_{2}, \theta ; \tilde{\epsilon}^{\prime}, \tilde{z}^{\prime}\right) \geq(\leq) S_{+}\left(\mu_{1}, \alpha, \mu_{2}, \theta ; \tilde{\epsilon}, \tilde{z}\right)
$$

and

$$
S_{-}\left(\mu_{1}, \alpha, \mu_{2}, \theta ; \tilde{\epsilon}^{\prime}, \tilde{z}^{\prime}\right) \geq(\leq) S_{-}\left(\mu_{1}, \alpha, \mu_{2}, \theta ; \tilde{\epsilon}, \tilde{z}\right)
$$

for $\left(\mu_{1}, \alpha, \mu_{2}, \theta\right) \in\left(R \times R_{+}\right)^{2}$.

Proof of Lemma 5. For $j=1,2,3$, letting $U_{j^{+}}\left(\mu_{1}, \alpha, \mu_{2} ; \tilde{\epsilon}, \tilde{z}\right)$ and $U_{j^{-}}\left(\mu_{1}, \alpha, \mu_{2} ; \tilde{\epsilon}, \tilde{z}\right)$ denote re- 
spectively the right and left partial derivatives of $U\left(\mu_{1}, \alpha, \mu_{2} ; \tilde{\epsilon}, \tilde{z}\right)$ with respect to the $j$ th argument of the function, for $\left(\mu_{1}, \alpha, \mu_{2}, \theta\right) \in\left(R \times R_{+}\right)^{2}$, we can write

$$
\begin{aligned}
& S_{+}\left(\mu_{1}, \alpha, \mu_{2}, \theta ; \tilde{\epsilon}, \tilde{z}\right) \\
& \quad= \begin{cases}-\frac{U_{2^{+}}\left(\mu_{1}, \alpha, \mu_{2} ; \tilde{\epsilon}, \tilde{z}\right)-\theta U_{3^{-}}\left(\mu_{1}, \alpha, \mu_{2} ; \tilde{\epsilon}, \tilde{z}\right)}{U_{1^{+}}\left(\mu_{1}, \alpha, \mu_{2} ; \tilde{\epsilon}, \tilde{z}\right)} & \text { if } U_{2^{+}}\left(\mu_{1}, \alpha, \mu_{2} ; \tilde{\epsilon}, \tilde{z}\right)-\theta U_{3^{-}}\left(\mu_{1}, \alpha, \mu_{2} ; \tilde{\epsilon}, \tilde{z}\right) \leq 0 ; \\
-\frac{U_{2^{+}}\left(\mu_{1}, \alpha, \mu_{2} ; \tilde{\epsilon}, \tilde{z}\right)-\theta U_{3^{-}}\left(\mu_{1}, \alpha, \mu_{2} ; \tilde{\epsilon}, \tilde{z}\right)}{U_{1^{-}}\left(\mu_{1}, \alpha, \mu_{2} ; \tilde{\epsilon}, \tilde{z}\right)} & \text { if } U_{2^{+}}\left(\mu_{1}, \alpha, \mu_{2} ; \tilde{\epsilon}, \tilde{z}\right)-\theta U_{3^{-}}\left(\mu_{1}, \alpha, \mu_{2} ; \tilde{\epsilon}, \tilde{z}\right) \geq 0 .\end{cases}
\end{aligned}
$$

and $S_{-}\left(\mu_{1}, \alpha, \mu_{2}, \theta ; \tilde{\epsilon}, \tilde{z}\right)$

$$
= \begin{cases}-\frac{U_{2^{-}}\left(\mu_{1}, \alpha, \mu_{2} ; \tilde{\epsilon}, \tilde{z}\right)-\theta U_{3}\left(\mu_{1}, \alpha, \mu_{2} ; \tilde{\epsilon}, \tilde{z}\right)}{U_{1-}\left(\mu_{1}, \alpha, \mu_{2} ; \tilde{\epsilon}, \tilde{z}\right)} & \text { if } U_{2^{-}}\left(\mu_{1}, \alpha, \mu_{2} ; \tilde{\epsilon}, \tilde{z}\right)-\theta U_{3^{+}}\left(\mu_{1}, \alpha, \mu_{2} ; \tilde{\epsilon}, \tilde{z}\right) \leq 0 \\ -\frac{U_{2^{-}}\left(\mu_{1}, \alpha, \mu_{2} ; \tilde{\epsilon}, \tilde{z}\right)-\theta U_{3}\left(\mu_{1}, \alpha, \mu_{2} ; \tilde{\epsilon}, \tilde{z}\right)}{U_{1+}\left(\mu_{1}, \alpha, \mu_{2} ; \tilde{\epsilon}, \tilde{z}\right)} & \text { if } U_{2^{-}}\left(\mu_{1}, \alpha, \mu_{2} ; \tilde{\epsilon}, \tilde{z}\right)-\theta U_{3^{+}}\left(\mu_{1}, \alpha, \mu_{2} ; \tilde{\epsilon}, \tilde{z}\right) \geq 0 .\end{cases}
$$

Letting $\tilde{w}=\mu_{w}+\tilde{z}$ and $\tilde{x}=\mu_{x}+\tilde{\epsilon}$ and $V_{q^{+}}\left(F_{\tilde{w}+q \tilde{x}, \Gamma-\beta q}\right)$ and $V_{q^{-}}\left(F_{\tilde{w}+q \tilde{x}, \Gamma-\beta q}\right)$ denote respectively the right and left derivatives of $V\left(F_{\tilde{w}+q \tilde{x}}, \Gamma-\beta q\right)$ with respect to $q$, under C-diversification, the optimal $q^{*}(\tilde{x}, \tilde{w}, \Gamma, \beta)$ that maximizes $V\left(F_{\tilde{w}+q \tilde{x}, \Gamma-\beta q}\right)=U\left(\mu_{w}+\mu_{x} q, q, \Gamma-\beta q ; \tilde{z}, \tilde{\epsilon}\right)$ is characterized by the following first-order conditions:

$$
\begin{aligned}
& V_{q^{+}}\left(F_{\tilde{w}+q^{*} \tilde{x}, \Gamma-\beta q^{*}}\right)=\mu_{x} U_{1^{+}}\left(\mu_{w}+\mu_{x} q^{*}, q^{*}, \Gamma-\beta q^{*} ; \tilde{\epsilon}, \tilde{z}\right) \\
& \quad+U_{2^{+}}\left(\mu_{w}+\mu_{x} q^{*}, q^{*}, \Gamma-\beta q^{*} ; \tilde{\epsilon}, \tilde{z}\right)-\beta U_{3^{-}}\left(\mu_{w}+\mu_{x} q^{*}, q^{*}, \Gamma-\beta q^{*} ; \tilde{\epsilon}, \tilde{z}\right) \leq 0
\end{aligned}
$$

and

$$
\begin{aligned}
& V_{q^{-}}\left(F_{\tilde{w}+q^{*} \tilde{x}, \Gamma-\beta q^{*}}\right)=\mu_{x} U_{1^{-}}\left(\mu_{w}+\mu_{x} q^{*}, q^{*}, \Gamma-\beta q^{*} ; \tilde{\epsilon}, \tilde{z}\right) \\
& \quad+U_{2^{-}}\left(\mu_{w}+\mu_{x} q^{*}, q^{*}, \Gamma-\beta q^{*} ; \tilde{\epsilon}, \tilde{z}\right)-\beta U_{3^{+}}\left(\mu_{w}+\mu_{x} q^{*}, q^{*}, \Gamma-\beta q^{*} ; \tilde{\epsilon}, \tilde{z}\right) \geq 0
\end{aligned}
$$

if $\mu_{x} \geq 0$, and by

$$
\begin{aligned}
& V_{q^{+}}\left(F_{\tilde{w}+q^{*} \tilde{x}, \Gamma-\beta q^{*}}\right)=\mu_{x} U_{1^{-}}\left(\mu_{w}+\mu_{x} q^{*}, q^{*}, \Gamma-\beta q^{*} ; \tilde{\epsilon}, \tilde{z}\right) \\
& \quad+U_{2^{+}}\left(\mu_{w}+\mu_{x} q^{*}, q^{*}, \Gamma-\beta q^{*} ; \tilde{\epsilon}, \tilde{z}\right)-\beta U_{3^{-}}\left(\mu_{w}+\mu_{x} q^{*}, q^{*}, \Gamma-\beta q^{*} ; \tilde{\epsilon}, \tilde{z}\right) \leq 0
\end{aligned}
$$

and

$$
\begin{aligned}
& V_{q^{-}}\left(F_{\tilde{w}+q^{*} \tilde{x}, \Gamma-\beta q^{*}}\right)=\mu_{x} U_{1^{+}}\left(\mu_{w}+\mu_{x} q^{*}, \Gamma-\beta q^{*}, q^{*} ; \tilde{\epsilon}, \tilde{z}\right) \\
& \quad+U_{2^{-}}\left(\mu_{w}+\mu_{x} q^{*}, q^{*}, \Gamma-\beta q^{*} ; \tilde{\epsilon}, \tilde{z}\right)-\beta U_{3^{+}}\left(\mu_{w}+\mu_{x} q^{*}, q^{*}, \Gamma-\beta q^{*} ; \tilde{\epsilon}, \tilde{z}\right) \geq 0
\end{aligned}
$$

if $\mu_{x}<0$.

If $\mu_{x} \geq 0$, which necessarily entails

$$
U_{2^{+}}\left(\mu_{w}+\mu_{x} q^{*}, q^{*}, \Gamma-\beta q^{*} ; \tilde{\epsilon}, \tilde{z}\right)-\beta U_{3^{-}}\left(\mu_{w}+\mu_{x} q^{*}, q^{*}, \Gamma-\beta q^{*} ; \tilde{\epsilon}, \tilde{z}\right)<0
$$

in $(3)$, or $\mu_{x}<0$ and $U_{2^{+}}\left(\mu_{w}+\mu_{x} q^{*}, q^{*}, \Gamma-\beta q^{*} ; \tilde{\epsilon}, \tilde{z}\right)-\beta U_{3^{-}}\left(\mu_{w}+\mu_{x} q^{*}, q^{*}, \Gamma-\beta q^{*} ; \tilde{\epsilon}, \tilde{z}\right) \geq 0$ in 
(5), then since (3) or (5) can be rewritten as

$$
\begin{gathered}
S_{+}\left(\mu_{w}+\mu_{x} q^{*}, \Gamma-\beta q^{*}, q^{*}, \beta ; \tilde{\epsilon}, \tilde{z}\right) \geq \mu_{x}, \\
S_{+}\left(\mu_{w}+\mu_{x} q^{*}, \Gamma-\beta q^{*}, q^{*}, \beta ; \tilde{\epsilon}^{\prime}, \tilde{z}^{\prime}\right) \geq S_{+}\left(\mu_{w}+\mu_{x} q^{*}, \Gamma-\beta q^{*}, q^{*}, \beta ; \tilde{\epsilon}, \tilde{z}\right) \geq \mu_{x}
\end{gathered}
$$

rules out $q^{*}\left(\mu_{x}+\tilde{\epsilon}^{\prime}, \mu_{w}+\tilde{z}^{\prime}, \Gamma, \beta\right)>q^{*}(\tilde{x}, \tilde{w}, \Gamma, \beta)$.

If $\mu_{x}<0$ and $U_{2^{+}}\left(\mu_{w}+\mu_{x} q^{*}, q^{*}, \Gamma-\beta q^{*} ; \tilde{\epsilon}, \tilde{z}\right)-\beta U_{3^{-}}\left(\mu_{w}+\mu_{x} q^{*}, q^{*}, \Gamma-\beta q^{*} ; \tilde{\epsilon}, \tilde{z}\right)<0$, since

$$
\begin{gathered}
S_{+}\left(\mu_{w}+\mu_{x} q^{*}, q^{*}, \Gamma-\beta q^{*}, \beta ; \tilde{\epsilon}^{\prime}, \tilde{z}^{\prime}\right) \\
=-\frac{U_{2^{+}}\left(\mu_{w}+\mu_{x} q^{*}, q^{*}, \Gamma-\beta q^{*} ; \tilde{\epsilon}^{\prime}, \tilde{z}^{\prime}\right)+\beta U_{3^{-}}\left(\mu_{w}+\mu_{x} q^{*}, q^{*}, \Gamma-\beta q^{*} ; \tilde{\epsilon}^{\prime}, \tilde{z}^{\prime}\right)}{U_{1^{+}}\left(\mu_{w}+\mu_{x} q^{*}, \Gamma-\beta q^{*}, q^{*} ; \tilde{\epsilon}^{\prime}, \tilde{z}^{\prime}\right)} \\
\geq S_{+}\left(\mu_{w}+\mu_{x} q^{*}, q^{*}, \Gamma-\beta q^{*}, \beta ; \tilde{\epsilon}, \tilde{z}\right) \\
=-\frac{U_{2^{+}}\left(\mu_{w}+\mu_{x} q^{*}, q^{*}, \Gamma-\beta q^{*}, \beta ; \tilde{\epsilon}, \tilde{z}\right)-\beta U_{3^{-}}\left(\mu_{w}+\mu_{x} q^{*}, q^{*}, \Gamma-\beta q^{*} ; \tilde{\epsilon}, \tilde{z}\right)}{U_{1^{+}}\left(\mu_{w}+\mu_{x} q^{*}, q^{*}, \Gamma-\beta q^{*} ; \tilde{\epsilon}, \tilde{z}\right)}>0,
\end{gathered}
$$

we have

$$
U_{2^{+}}\left(\mu_{w}+\mu_{x} q^{*}, q^{*}, \Gamma-\beta q^{*} ; \tilde{\epsilon}^{\prime}, \tilde{z}^{\prime}\right)-\beta U_{3^{-}}\left(\mu_{w}+\mu_{x} q^{*}, q^{*}, \Gamma-\beta q^{*} ; \tilde{\epsilon}^{\prime}, \tilde{z}^{\prime}\right)<0
$$

Thus

$$
\begin{aligned}
& V_{q^{+}}\left(F_{\tilde{w}^{\prime}+q^{*} \tilde{x}^{\prime}, \Gamma-\beta q^{*}}\right)=\mu_{x} U_{1^{-}}\left(\mu_{w}+\mu_{x} q^{*}, q^{*}, \Gamma-\beta q^{*} ; \tilde{\epsilon}^{\prime}, \tilde{z}^{\prime}\right) \\
& \quad+U_{2^{+}}\left(\mu_{w}+\mu_{x} q^{*}, q^{*}, \Gamma-\beta q^{*} ; \tilde{\epsilon}^{\prime}, \tilde{z}^{\prime}\right)-\beta U_{3^{-}}\left(\mu_{w}+\mu_{x} q^{*}, q^{*}, \Gamma-\beta q^{*} ; \tilde{\epsilon}^{\prime}, \tilde{z}^{\prime}\right)<0
\end{aligned}
$$

which rules out $q^{*}\left(\mu_{x}+\tilde{\epsilon}^{\prime}, \mu_{w}+\tilde{z}^{\prime}, \Gamma, \beta\right)>q^{*}(\tilde{x}, \tilde{w}, \Gamma, \beta)$.

Noting that $S_{+}\left(\mu_{1}, \alpha, \mu_{2}, \theta ; \tilde{\epsilon}^{\prime}, \tilde{z}^{\prime}\right) \geq S_{+}\left(\mu_{1}, \alpha, \mu_{2}, \theta ; \tilde{\epsilon}, \tilde{z}\right)$ for $\left(\mu_{1}, \alpha, \mu_{2}, \theta\right) \in\left(R \times R_{+}\right)^{2}$ implies and is implied by $S_{-}\left(\mu_{1}, \alpha, \mu_{2}, \theta ; \tilde{\epsilon}^{\prime}, \tilde{z}^{\prime}\right) \geq S_{-}\left(\mu_{1}, \alpha, \mu_{2}, \theta ; \tilde{\epsilon}, \tilde{z}\right)$ for $\left(\mu_{1}, \alpha, \mu_{2}, \theta\right) \in\left(R \times R_{+}\right)^{2}$, to prove the converse, suppose there exist $\left(\hat{\mu}_{1}, \hat{\alpha}, \hat{\mu}_{2}, \hat{\theta}\right) \in\left(R \times R_{+}\right)^{2}$ such that

$$
\begin{array}{r}
S_{+}\left(\hat{\mu}_{1}, \hat{\alpha}, \hat{\mu}_{2}, \hat{\theta} ; \tilde{\epsilon}^{\prime}, \tilde{z}^{\prime}\right)<S_{+}\left(\hat{\mu}_{1}, \hat{\alpha}, \hat{\mu} 2, \hat{\theta} ; \tilde{\epsilon}, \tilde{z}\right) \\
S_{-}\left(\hat{\mu}_{1}, \hat{\alpha}, \hat{\mu}_{2}, \hat{\theta} ; \tilde{\epsilon}^{\prime}, \tilde{z}^{\prime}\right)<S_{-}\left(\hat{\mu}_{1}, \hat{\alpha}, \hat{\mu}_{2}, \hat{\theta} ; \tilde{\epsilon}, \tilde{z}\right) .
\end{array}
$$

and

Since we can always find $\hat{\mu}_{x}, \hat{\mu}_{w}$, and $\hat{\Gamma}$ such that $\hat{\mu}_{w}+\hat{\alpha} \hat{\mu}_{x}=\hat{\mu}_{1}, \hat{\Gamma}-\hat{\alpha} \hat{\theta}=\hat{\mu}_{2}$ and

and

$$
\begin{gathered}
\hat{S}_{+}\left(\hat{\mu}_{1}, \hat{\alpha}, \hat{\mu}_{2}, \hat{\theta} ; \tilde{\epsilon}, \tilde{z}\right)=\hat{\mu}_{x} \\
\hat{S}_{-}\left(\hat{\mu}_{1}, \hat{\alpha}, \hat{\mu}_{2}, \hat{\theta} ; \tilde{\epsilon}, \tilde{z}\right) \leq \hat{\mu}_{x},
\end{gathered}
$$

define $\tilde{x} \equiv \hat{\mu}_{x}+\tilde{\epsilon}, \tilde{x}^{\prime} \equiv \hat{\mu}_{x}+\tilde{\epsilon}^{\prime}, \tilde{w} \equiv \hat{\mu}_{w}+\tilde{z}$, and $\tilde{w}^{\prime} \equiv \hat{\mu}_{w}+\tilde{z}^{\prime}$. If $\hat{\mu}_{x} \geq 0$, (9) is equivalent to

$$
V_{q^{+}}\left(F_{\tilde{w}+\hat{\alpha} \tilde{x}, \hat{\Gamma}-\hat{\theta} \hat{\alpha}}\right)=\hat{\mu}_{x} U_{1^{+}}\left(\hat{\mu}_{1}, \hat{\alpha}, \hat{\mu}_{2} ; \tilde{\epsilon}, \tilde{z}\right)+U_{2^{+}}\left(\hat{\mu}_{1}, \hat{\alpha}, \hat{\mu}_{2} ; \tilde{\epsilon}, \tilde{z}\right)-\hat{\theta} U_{3^{-}}\left(\hat{\mu}_{1}, \hat{\alpha}, \hat{\mu} 2 ; \tilde{\epsilon}, \tilde{z}\right)=0
$$


and (10) implies

$$
V_{q^{-}}\left(F_{\tilde{w}+\hat{\alpha} \tilde{x}, \hat{\Gamma}-\hat{\theta} \hat{\alpha}}\right)=\hat{\mu}_{x} U_{1^{-}}\left(\hat{\mu}_{1}, \hat{\alpha}, \hat{\mu}_{2} ; \tilde{\epsilon}, \tilde{z}\right)+U_{2^{-}}\left(\hat{\mu}_{1}, \hat{\alpha}, \hat{\mu}_{2} ; \tilde{\epsilon}, \tilde{z}\right)-\hat{\theta} U_{3^{+}}\left(\hat{\mu}_{1}, \hat{\alpha}, \hat{\mu}_{2} ; \tilde{\epsilon}, \tilde{z}\right) \geq 0
$$

hence $\hat{\alpha}=q^{*}(\tilde{x}, \tilde{w}, \hat{\Gamma}, \hat{\theta})$. (7) then implies

$$
\begin{aligned}
& V_{q^{+}}\left(F_{\tilde{w}^{\prime}+\hat{\alpha} \tilde{x}^{\prime}, \hat{\Gamma}-\hat{\theta} \hat{\alpha}}\right) \\
& \quad=\hat{\mu}_{x} U_{1^{+}}\left(\hat{\mu}_{1}, \hat{\alpha}, \hat{\mu}_{2} ; \tilde{\epsilon}^{\prime}, \tilde{z}^{\prime}\right)+U_{2^{+}}\left(\hat{\mu}_{1}, \hat{\alpha}, \hat{\mu}_{2} ; \tilde{\epsilon}^{\prime}, \tilde{z}^{\prime}\right)-\hat{\theta} U_{3^{-}}\left(\hat{\mu}_{1}, \hat{\alpha}, \hat{\mu}_{2} ; \tilde{\epsilon}^{\prime}, \tilde{z}^{\prime}\right)>0
\end{aligned}
$$

and (8) implies

$$
\begin{aligned}
& V_{q^{-}}\left(F_{\tilde{w}^{\prime}+\hat{\alpha} \tilde{x}^{\prime}, \hat{\Gamma}-\hat{\theta} \hat{\alpha}}\right) \\
& \quad=\hat{\mu}_{x} U_{1^{-}}\left(\hat{\mu}_{1}, \hat{\alpha}, \hat{\mu}_{2} ; \tilde{\epsilon}^{\prime}, \tilde{z}^{\prime}\right)+U_{2^{-}}\left(\hat{\mu}_{1}, \hat{\alpha}, \hat{\mu}_{2} ; \tilde{\epsilon}^{\prime}, \tilde{z}^{\prime}\right)-\hat{\theta} U_{3^{+}}\left(\hat{\mu}_{1}, \hat{\mu}_{2}, \hat{\alpha}, \hat{\theta} ; \tilde{\epsilon}^{\prime}, \tilde{z}_{d}^{\prime}\right)>0
\end{aligned}
$$

since if

$$
U_{2^{-}}\left(\hat{\mu}_{1}, \hat{\alpha}, \hat{\mu}_{2} ; \tilde{\epsilon}, \tilde{z}\right)-\hat{\theta} U_{3^{+}}\left(\hat{\mu}_{1}, \hat{\alpha}, \hat{\mu}_{2} ; \tilde{\epsilon}, \tilde{z}\right) \leq 0 \text { and } U_{2^{-}}\left(\hat{\mu}_{1}, \hat{\alpha}, \hat{\mu}_{2} ; \tilde{\epsilon}^{\prime}, \tilde{z}^{\prime}\right)-\hat{\theta} U_{3^{+}}\left(\hat{\mu}_{1}, \hat{\alpha}, \hat{\mu}_{2} ; \tilde{\epsilon}^{\prime}, \tilde{z}^{\prime}\right) \leq 0
$$

(8) and (10) imply

$$
S_{-}\left(\hat{\mu}_{1}, \hat{\alpha}, \hat{\mu}_{2}, \hat{\theta} ; \tilde{\epsilon}^{\prime}, \tilde{z}^{\prime}\right)<\hat{\mu}_{x}
$$

which is equivalent to $(12)$ and if $U_{2^{-}}\left(\hat{\mu}_{1}, \hat{\alpha}, \hat{\mu}_{2} ; \tilde{\epsilon}, \tilde{z}\right)-\hat{\theta} U_{3^{+}}\left(\hat{\mu}_{1}, \hat{\alpha}, \hat{\mu}_{2} ; \tilde{\epsilon}, \tilde{z}\right)>0$

$$
\begin{aligned}
& S_{-}\left(\hat{\mu}_{1}, \hat{\alpha}, \hat{\mu}_{2}, \hat{\theta} ; \tilde{\epsilon}^{\prime}, \tilde{z}^{\prime}\right)=-\frac{U_{2^{-}}\left(\hat{\mu}_{1}, \hat{\alpha}, \hat{\mu}_{2} ; \tilde{\epsilon}^{\prime}, \tilde{z}^{\prime}\right)-\hat{\theta} U_{3^{+}}\left(\hat{\mu}_{1}, \hat{\alpha}, \hat{\mu}_{2} ; \tilde{\epsilon}^{\prime}, \tilde{z}^{\prime}\right)}{U_{1^{+}}\left(\hat{\mu}_{1}, \hat{\alpha}, \hat{\mu}_{2} ; \tilde{\epsilon}^{\prime}, \tilde{z}^{\prime}\right)} \\
< & S_{-}\left(\hat{\mu}_{1}, \hat{\alpha}, \hat{\mu}_{2}, \hat{\theta} ; \tilde{\epsilon}, \tilde{z}\right)=-\frac{U_{2^{-}}\left(\hat{\mu}_{1}, \hat{\alpha}, \hat{\mu}_{2} ; \tilde{\epsilon}, \tilde{z}\right)-\hat{\theta} U_{3^{+}}\left(\hat{\mu}_{1}, \hat{\alpha}, \hat{\mu}_{2} ; \tilde{\epsilon}, \tilde{z}\right)}{U_{1^{+}}\left(\hat{\mu}_{1}, \hat{\alpha}, \hat{\mu}_{2} ; \tilde{\epsilon}, \tilde{z}\right)}<0,
\end{aligned}
$$

which gives

$$
U_{2^{-}}\left(\hat{\mu}_{1}, \hat{\alpha}, \hat{\mu}_{2} ; \tilde{\epsilon}^{\prime}, \tilde{z}^{\prime}\right)-\hat{\theta} U_{3^{+}}\left(\hat{\mu}_{1}, \hat{\alpha}, \hat{\mu}_{2} ; \tilde{\epsilon}^{\prime}, \tilde{z}^{\prime}\right)>0 .
$$

Thus $q^{*}(\tilde{x}, \tilde{w}, \hat{\Gamma}, \hat{\theta})<q^{*}\left(\tilde{x}^{\prime}, \tilde{w}^{\prime}, \hat{\Gamma}, \hat{\theta}\right)$. If $\mu_{\tilde{x}}<0$, since (9) and (10) in this case entail that

$$
U_{2^{+}}\left(\hat{\mu}_{1}, \hat{\alpha}, \hat{\mu}_{2} ; \tilde{\epsilon}, \tilde{z}\right)-\hat{\theta} U_{3^{-}}\left(\hat{\mu}_{1}, \hat{\alpha}, \hat{\mu}_{2} ; \tilde{\epsilon}, \tilde{z}\right)>0 \text { and } U_{2^{-}}\left(\hat{\mu}_{1}, \hat{\alpha}, \hat{\mu}_{2} ; \tilde{\epsilon}, \tilde{z}\right)-\hat{\theta} U_{3^{+}}\left(\hat{\mu}_{1}, \hat{\alpha}, \hat{\mu} 2 ; \tilde{\epsilon}, \tilde{z}\right)>0
$$

we first also have $\hat{\alpha}=q^{*}(\tilde{x}, \tilde{w}, \hat{\Gamma}, \hat{\theta})$ and (7)—(10) imply

$$
V_{q^{+}}\left(F_{\tilde{w}^{\prime}+\hat{\alpha} \tilde{x}^{\prime}, \hat{\Gamma}-\hat{\theta} \hat{\alpha}}\right)=\hat{\mu}_{x} U_{1^{-}}\left(\hat{\mu}_{1}, \hat{\alpha}, \hat{\mu}_{2} ; \tilde{\epsilon}^{\prime}, \tilde{z}^{\prime}\right)+U_{2^{+}}\left(\hat{\mu}_{1}, \hat{\alpha}, \hat{\mu}_{2} ; \tilde{\epsilon}^{\prime}, \tilde{z}^{\prime}\right)-\hat{\theta} U_{3^{-}}\left(\hat{\mu}_{1}, \hat{\alpha}, \hat{\mu}_{2} ; \tilde{\epsilon}^{\prime}, \tilde{z}^{\prime}\right)>0
$$

and

$$
V_{q^{-}}\left(F_{\tilde{w}^{\prime}+\hat{\alpha} \tilde{x}^{\prime}, \hat{\Gamma}-\hat{\theta} \hat{\alpha}}\right)=\hat{\mu}_{x} U_{1^{+}}\left(\hat{\mu}_{1}, \hat{\alpha}, \hat{\mu}_{2} ; \tilde{\epsilon}^{\prime}, \tilde{z}^{\prime}\right)+U_{2^{-}}\left(\hat{\mu}_{1}, \hat{\alpha}, \hat{\mu}_{2} ; \tilde{\epsilon}^{\prime}, \tilde{z}^{\prime}\right)-\hat{\theta} U_{3^{+}}\left(\hat{\mu}_{1}, \hat{\alpha}, \hat{\mu}_{2} ; \tilde{\epsilon}^{\prime}, \tilde{z}^{\prime}\right)>0
$$

which gives $q^{*}(\tilde{x}, \tilde{w}, \hat{\Gamma}, \hat{\theta})<q^{*}\left(\tilde{x}^{\prime}, \tilde{w}^{\prime}, \hat{\Gamma}, \hat{\theta}\right)$. 


\section{A2. Characterization of Prudence and Judicious Appetite for Risk in the EU framework}

We characterize the preference conditions of $N$ th-degree prudence and $N$ th-degree judicious appetite for (aggravated) risk in the EU framework, which serves to give an indication of the strength of these conditions and to relate the results on them obtained in our general framework to existing results. For $n, m=1,2, \ldots, u_{(n)(m)}(x, y)$ denotes the $n$th partial derivative with respect to $x$ of the $m$ th partial derivative of $u(x, y)$ with respect to $y$, and $u_{(n)(0)}(x, y)$ and $u_{(0)(n)}(x, y)$ denote the $n$th partial derivatives of $u(x, y)$ with respect to $x$ and $y$ respectively. Our notion of prudence can be characterized in the EU framework as follows.

Proposition 4 In the EU framework, suppose $u_{(0)(1)}(\cdot, \cdot) \geq 0$ and $u_{(1)(0)}(\cdot, \cdot) \geq 0$.

(i) Assuming $(-1)^{N} u_{(N)(1)}(\cdot, \cdot) \leq 0$, exhibits $N$ th-degree prudence if $(-1)^{N+1} u_{(N+1)(0)}(\cdot, \cdot)$ $\geq 0$.

(ii) Assuming $(-1)^{N} u_{(N)(1)}(\cdot, \cdot) \geq 0$, exhibits $N$ th-degree prudence only if $(-1)^{N+1} u_{(N+1)(0)}(\cdot, \cdot) \geq 0$.

\section{Proof.}

(i) By Lemma $2,(-1)^{N+1} u_{(N+1)(0)}(\cdot, \cdot) \geq 0$ if and only if for $\tilde{w}^{\prime}$ being an $N$ th-degree risk increase of $\tilde{w}$,

$$
E u_{(1)(0)}(\tilde{w}, y) \leq E u_{(1)(0)}\left(\tilde{w}^{\prime}, y\right),
$$

which in turn implies and is implied by

$$
E u(\tilde{w}+\pi, y)-E u(\tilde{w}, y) \leq E u\left(\tilde{w}^{\prime}+\pi, y\right)-E u\left(\tilde{w}^{\prime}, y\right) \text { for all } y \in[a, b] \text { and } \pi>0 .
$$

Rearranging, we have

$$
E u(\tilde{w}+\pi, y)-E u\left(\tilde{w}^{\prime}+\pi, y\right) \leq E u(\tilde{w}, y)-E u\left(\tilde{w}^{\prime}, y\right) .
$$

Since $(-1)^{N} u_{(N)(1)}() \leq$,0 implies for $\delta>0$

$$
E u(\tilde{w}+\pi, y-\delta)-E u\left(\tilde{w}^{\prime}+\pi, y-\delta\right) \leq E u(\tilde{w}+\pi, y)-E u\left(\tilde{w}^{\prime}+\pi, y\right),
$$

we have

$$
E u(\tilde{w}+\pi, y-\delta)-E u\left(\tilde{w}^{\prime}+\pi, y-\delta\right) \leq E u(\tilde{w}, y)-E u\left(\tilde{w}^{\prime}, y\right)
$$

and thus $E u(\tilde{w}+\pi, y-\delta)=E u(\tilde{w}, y)$ implies $E u\left(\tilde{w}^{\prime}+\pi, y-\delta\right) \geq E u\left(\tilde{w}^{\prime}, y\right)$ or $\succsim$ exhibits $N$ thdegree prudence.

(ii) For $\delta>0$, let $\hat{\pi}=\pi(\delta)$ be such that

$$
E u(\tilde{w}+\hat{\pi}, y-\delta)=E u(\tilde{w}, y)
$$


Given $u_{(0)(1)}(\cdot, \cdot) \geq 0$ and $u_{(1)(0)}(\cdot, \cdot) \geq 0, \hat{\pi} \geq 0$. In the EU framework, $\succsim$ exhibiting $N$ th-degree prudence then implies

$$
E u\left(\tilde{w}^{\prime}+\hat{\pi}, y-\delta\right) \geq E u\left(\tilde{w}^{\prime}, y\right)
$$

where $F_{\tilde{w}^{\prime}}$ is an Nth-degree risk increase of $F_{\tilde{w}}$. We thus have

$$
E u(\tilde{w}+\hat{\pi}, y-\delta)-E u\left(\tilde{w}^{\prime}+\hat{\pi}, y-\delta\right) \leq E u(\tilde{w}, y)-E u\left(\tilde{w}^{\prime}, y\right) .
$$

Since (by Lemma 2) $(-1)^{N} u_{(N)(1)}() \geq$,0 implies $E u_{(0)(1)}(\tilde{w}+\hat{\pi}, y)-E u_{(0)(1)}\left(\tilde{w}^{\prime}+\hat{\pi}, y\right) \leq 0$, for $\delta>0$,

$$
E u(\tilde{w}+\hat{\pi}, y-\delta)-E u\left(\tilde{w}^{\prime}+\hat{\pi}, y-\delta\right) \geq E u(\tilde{w}+\hat{\pi}, y)-E u\left(\tilde{w}^{\prime}+\hat{\pi}, y\right)
$$

Hence

$$
E u(\tilde{w}+\hat{\pi}, y)-E u\left(\tilde{w}^{\prime}+\hat{\pi}, y\right) \leq E u(\tilde{w}, y)-E u\left(\tilde{w}^{\prime}, y\right)
$$

or equivalently

$$
E u(\tilde{w}+\hat{\pi}, y)-E u(\tilde{w}, y) \leq E u\left(\tilde{w}^{\prime}+\hat{\pi}, y\right)-E u\left(\tilde{w}^{\prime}, y\right)
$$

which holds for all $\hat{\pi} \geq 0$ if and only if $E u_{(1)(0)}(\tilde{w}, y) \leq E u_{(1)(0)}\left(\tilde{w}^{\prime}, y\right)$, which (by Lemma 2) implies and is implied by $(-1)^{N+1} u_{(N+1)(0)}(\cdot, \cdot) \geq 0$.

Thus assuming a time-separable VNM utility function that gives $(-1)^{N} u_{(N)(1)}(\cdot, \cdot)=0$, and $N=2, \succsim$ exhibits (2nd-degree) prudence if and only if $u_{(3)(0)}(\cdot, \cdot) \geq 0$, which was first shown by Leland (1968) to imply a precautionary saving motive. Eeckhoudt and Schlesinger (2008) generalize Leland's (1968) result by showing that, assuming time-separability, $(-1)^{N+1} u_{(N+1)(0)}(\cdot, \cdot) \geq 0$ is sufficient for the amount of saving to rise in response to an $N$ th-degree risk increase in future income.

The next result characterizes the preference conditions of judicious appetite for risk and judicious appetite for aggravated risk in the EU framework.

Proposition 5 In the EU framework, suppose $u_{(0)(1)}(\cdot, \cdot) \geq 0$ and $u_{(1)(0)}(\cdot, \cdot) \geq 0$.

(i) If $(-1)^{n} u_{(n)(0)}(\cdot, \cdot) \leq 0$ for $n=N, N+1$ and $(-1)^{N} u_{(N)(1)}(\cdot, \cdot) \geq 0$ and

$$
-x \frac{u_{(N+1)(0)}(w+x, y)}{u_{(N)(0)}(w+x, y)} \leq N \text { for all } x, w, y
$$

then $\succsim$ exhibits $N$ th-degree judicious appetite for aggravated risk.

(ii) Assuming $(-1)^{n} u_{(n)(0)}(\cdot, \cdot) \leq 0$ for $n=N, N+1$ and $(-1)^{N} u_{(N)(1)}(\cdot, \cdot) \leq 0$, (13) holds if $\succsim$ exhibits $N$ th-degree judicious appetite for aggravated risk with $\tilde{\epsilon}$ being positive expectation 
dependent on $\tilde{w}$.

Proof. We first show that, for $\alpha_{2}>\alpha_{1}>0$ and $F_{\tilde{x}^{\prime} \mid \tilde{w}}$ being an $N$ th-degree risk increase of $F_{\tilde{x} \mid \tilde{w}}$,

$$
E u\left(\tilde{w}+\alpha_{1} \tilde{x}, y\right)-E u\left(\tilde{w}+\alpha_{1} \tilde{x}^{\prime}, y\right)-\left[E u\left(\tilde{w}+\alpha_{2} \tilde{x}, y\right)-E u\left(\tilde{w}+\alpha_{2} \tilde{x}^{\prime}, y\right)\right] \leq 0
$$

if and only if

$$
-x \frac{u_{(N+1)(0)}(w+x, y)}{u_{(N)(0)}(w+x, y)} \leq N \text { for all } x, w, y .
$$

Defining $Q(\alpha, w)=E[u(\tilde{w}+\alpha \tilde{x}, y) \mid \tilde{w}=w]-E\left[u\left(\tilde{w}+\alpha \tilde{x}^{\prime}, y\right) \mid \tilde{w}=w\right]$ and denoting the partial derivative of $Q$ respect to $\alpha$ by $Q_{\alpha}$, (14) can be further written as $E_{\tilde{w}}\left[Q\left(\alpha_{1}, \tilde{w}\right)-Q\left(\alpha_{2}, \tilde{w}\right)\right] \leq 0$, which is true (for all $\tilde{w}$ ) if and only if $Q_{\alpha}(\alpha, w) \geq 0$ for all $w$ and $\alpha \geq 0$.

Let $\phi(x, w, y)=x u_{(1)(0)}(w+\alpha x, y)$. By Lemma $2, Q_{\alpha}(\alpha, w)=E\left[\tilde{x} u_{(1)(0)}(\tilde{w}+\alpha \tilde{x}, y) \mid \tilde{w}=\right.$ $w]-E\left[\tilde{x}^{\prime} u_{(1)(0)}\left(\tilde{w}+\alpha \tilde{x}^{\prime}, y\right) \mid \tilde{w}=w\right]=E[\phi(\tilde{x}, \tilde{w}, y) \mid \tilde{w}=w]-E\left[\phi\left(\tilde{x}^{\prime}, \tilde{w}, y\right) \mid \tilde{w}=w\right] \geq 0$ for all $w$ and $\tilde{x}$ and $\tilde{x}^{\prime}$ such that $F_{\tilde{x}} \mid \tilde{w}$ is an $N$ th-degree risk increase of $F_{\tilde{x} \mid \tilde{w}}$ if and only if

$$
(-1)^{N} \frac{\partial^{N} \phi(x, w, y)}{\partial x^{N}} \leq 0 \text { for all } x, w, y
$$

which, given $(-1)^{n} u_{(n)(0)} \leq 0$ for $n=N, N+1$, is equivalent to

$$
-x \frac{u_{(N+1)(0)}(w+x, y)}{u_{(N)(0)}(w+x, y)} \leq N \text { for all } x, w, y .
$$

(i) Since $(-1)^{N} u_{(N)(1)}(\cdot, \cdot) \geq 0$ implies $E u_{(0)(1)}(\tilde{w}+\alpha \tilde{x}, y)-E u_{(0)(1)}\left(\tilde{w}+\alpha \tilde{x}^{\prime}, y\right) \leq 0$ where $\alpha>1$ and $F_{\tilde{x}^{\prime} \mid \tilde{w}}$ is an $N$ th-degree risk increase of $F_{\tilde{x} \mid \tilde{w}}$ and thus for $\delta>0$

$$
E u(\tilde{w}+\alpha \tilde{x}, y)-E u\left(\tilde{w}+\alpha \tilde{x}^{\prime}, y\right) \leq E u(\tilde{w}+\alpha \tilde{x}, y-\delta)-E u\left(\tilde{w}+\alpha \tilde{x}^{\prime}, y-\delta\right),
$$

(14) implies

$$
E u\left(\tilde{w}+\tilde{x}^{\prime}, y\right)-E u\left(\tilde{w}+\alpha \tilde{x}^{\prime}, y-\delta\right) \geq E u(\tilde{w}+\tilde{x}, y)-E u(\tilde{w}+\alpha \tilde{x}, y-\delta) .
$$

Suppose $F_{\tilde{w}_{0}+\tilde{\epsilon}, y} \sim F_{\tilde{w}_{0}+\pi+\alpha \tilde{\epsilon}, y-\delta}$. Letting $k=\pi /(\alpha-1)$ and $\tilde{w}_{0}=\tilde{w}+k$ and $\tilde{x}=\tilde{\epsilon}+k$, and $\tilde{x}_{d}=\tilde{\epsilon}_{d}+k$, since $\tilde{w}_{0}+\tilde{\epsilon}=\tilde{w}+k+\tilde{x}-k=\tilde{w}+\tilde{x}$ and $\tilde{w}_{0}+\pi+\alpha \tilde{\epsilon}=\tilde{w}+k+\pi+\alpha \tilde{x}-\alpha k=\tilde{w}+\alpha \tilde{x}$, we have $F_{\tilde{w}+\tilde{x}, y} \sim F_{\tilde{w}+\alpha \tilde{x}, y-\delta}$, which, by (15), implies $F_{\tilde{w}+\tilde{x}^{\prime}, y} \succsim F_{\tilde{w}+\alpha \tilde{x}^{\prime}, y-\delta}$ or equivalently $F_{\tilde{w}_{0}+\tilde{\epsilon}^{\prime}, y} \succsim$ $F_{\tilde{w}_{0}+\pi+\alpha \tilde{\epsilon}^{\prime}, y-\delta}\left(\right.$ since $\tilde{w}_{0}+\tilde{\epsilon}^{\prime}=\tilde{w}+k+\tilde{x}^{\prime}-k=\tilde{w}+\tilde{x}^{\prime}$ and $\left.\tilde{w}_{0}+\pi+\alpha \tilde{\epsilon}^{\prime}=\tilde{w}+k+\pi+\alpha \tilde{x}^{\prime}-\alpha k=\tilde{w}+\alpha \tilde{x}^{\prime}\right)$.

That is, $\succsim$ exhibits $N$ th-degree judicious appetite for aggravated risk.

(ii) For $\alpha>1$ and $\delta>0$, let $\hat{\pi}=\pi(\alpha, \delta)$ be such that

$$
E u(\tilde{w}+\tilde{\epsilon}, y)=E u(\tilde{w}+\hat{\pi}+\alpha \tilde{\epsilon}, y-\delta) .
$$

In the EU framework, $\succsim$ exhibiting $N$ th-degree judicious appetite for aggravated risk then implies 


$$
E u\left(\tilde{w}+\tilde{\epsilon}^{\prime}, y\right) \geq E u\left(\tilde{w}+\hat{\pi}+\alpha \tilde{\epsilon}^{\prime}, y-\delta\right)
$$

where $F_{\tilde{\epsilon}^{\prime} \mid \tilde{w}}$ is an Nth-degree risk increase of $F_{\tilde{\epsilon} \mid \tilde{w}}$. We thus have

$$
E u(\tilde{w}+\tilde{\epsilon}, y)-E u\left(\tilde{w}+\tilde{\epsilon}^{\prime}, y\right) \leq E u(\tilde{w}+\hat{\pi}+\alpha \tilde{\epsilon}, y-\delta)-E u\left(\tilde{w}+\hat{\pi}+\alpha \tilde{\epsilon}^{\prime}, y-\delta\right) .
$$

Since $(-1)^{N} u_{(N)(1)}() \leq$,0 implies $E u_{(0)(1)}(\tilde{w}+\hat{\pi}+\alpha \tilde{\epsilon}, y)-E u_{(0)(1)}\left(\tilde{w}+\hat{\pi}+\alpha \tilde{\epsilon}^{\prime}, y\right) \geq 0$, for $\delta>0$,

$$
E u(\tilde{w}+\hat{\pi}+\alpha \tilde{\epsilon}, y-\delta)-E u\left(\tilde{w}+\hat{\pi}+\alpha \tilde{\epsilon}^{\prime}, y-\delta\right) \leq E u(\tilde{w}+\hat{\pi}+\alpha \tilde{\epsilon}, y)-E u\left(\tilde{w}+\hat{\pi}+\alpha \tilde{\epsilon}^{\prime}, y\right) .
$$

Furthermore since $\hat{\pi} \geq 0$ under the assumption that $\tilde{\epsilon}$ is positive expectation dependent on $\tilde{w}$ and $E u_{(1)(0)}(\tilde{w}+\alpha \tilde{\epsilon}, y)-E u_{(1)(0)}\left(\tilde{w}+\alpha \tilde{\epsilon}^{\prime}, \tilde{y}\right) \leq 0$ for $F_{\tilde{\epsilon}^{\prime} \mid \tilde{w}}$ being an $N$ th-degree risk increase of $F_{\tilde{\epsilon} \mid \tilde{w}}$ under the assumption that $(-1)^{N+1} u_{(N+1)(0)}(x, y) \leq 0$ for all $x, y$, we have

$$
E u(\tilde{w}+\hat{\pi}+\alpha \tilde{\epsilon}, y)-E u\left(\tilde{w}+\hat{\pi}+\alpha \tilde{\epsilon}^{\prime}, y\right) \leq E u(\tilde{w}+\alpha \tilde{\epsilon}, y)-E u\left(\tilde{w}+\alpha \tilde{\epsilon}^{\prime}, y\right) .
$$

That is, we have, for $\alpha>1$

$$
E u(\tilde{w}+\tilde{\epsilon} y)-E u\left(\tilde{w}+\tilde{\epsilon}^{\prime}, y\right) \leq E u(\tilde{w}+\alpha \tilde{\epsilon}, y)-E u\left(\tilde{w}+\alpha \tilde{\epsilon}^{\prime}, y\right)
$$

which is equivalent to (14) assuming $E[\tilde{x}]=0$ (since, for $\alpha>0, F_{\alpha \tilde{\epsilon}^{\prime} \mid \tilde{w}}$ is an $N$ th-degree risk increase of $F_{\alpha \tilde{\epsilon} \mid \tilde{w}}$ if and only if $F_{\tilde{\epsilon}^{\prime} \mid \tilde{w}}$ is an $N$ th-degree risk increase of $\left.F_{\tilde{\epsilon} \mid \tilde{w}}\right)$ and thus by essentially the same proof can be shown to imply and be implied by

$$
-x \frac{u_{(N+1)(0)}(w+x, y)}{u_{(N)(0)}(w+x, y)} \leq N \text { for all } x, w, y
$$

Corollary 1 In the EU framework, suppose $u_{(0)(1)}(\cdot, \cdot) \geq 0$ and $u_{(1)(0)}(\cdot, \cdot) \geq 0$.

(i) Assuming $(-1)^{n} u_{(n)(0)}(\cdot, \cdot) \leq 0$ for $n=N, N+1$ and $(-1)^{N} u_{(N)(1)}(\cdot, \cdot)=0$, exhibits $N$ th-degree judicious appetite for aggravated risk with $\tilde{\epsilon}$ being positive expectation dependent on $\tilde{w}$ if and only if (13) holds.

(ii) Assuming $(-1)^{n} u_{(n)(0)}(\cdot, \cdot) \leq 0$ for $n=N, N+1$, if (13) holds, then $\succsim$ exhibits $N$ th-degree judicious appetite for risk.

(iii) Assuming $(-1)^{n} u_{(n)(0)}(\cdot, \cdot) \leq 0$ for $n=N, N+1$ and $\tilde{\epsilon}$, $\succsim$ exhibits $N$ th-degree judicious appetite for risk with $\tilde{\epsilon}$ being positive expectation dependent on $\tilde{w}$ if and only if (13) holds.

That is, in the EU framework, assuming $(-1)^{n} u_{(n)(0)}(\cdot, \cdot) \leq 0$ for $n=N, N+1$ (and $\left.(-1)^{N} u_{(N)(1)}(\cdot, \cdot) \geq 0\right)$, the condition (13) is sufficient for $N$ th-degree judicious appetite for 
(aggravated) risk. And under the assumption that $(-1)^{n} u_{(n)(0)}(\cdot, \cdot) \leq 0$ for $n=N, N+1$ (and $\left.(-1)^{N} u_{(N)(1)}(\cdot, \cdot)=0\right)$ and $\tilde{\epsilon}$ is positive expectation dependent on $\tilde{w},(13)$ is both necessary and sufficient for $N$ th-degree judicious appetite for (aggravated) risk. In view of Proposition 3 , these characterizations explain the role played by the condition (13) in the existing results obtained in the EU framework: Chiu and Eeckhoudt (2010) show in the standard labor supply model that assuming (13) along with $(-1)^{N} u_{(N)(1)}(\cdot, \cdot) \geq 0$ and $(-1)^{n} u_{(n)(0)}(\cdot, \cdot) \leq 0$ for $n=N, N+1$, labor supply decreases in response to an $N$ th-degree risk increase in the uncertain wage rate. Chiu, Eeckhoudt and Rey (2012) show in the two-asset portfolio choice model that (13) along with $(-1)^{n} u_{(n)(0)}(\cdot, \cdot) \leq 0$ for $n=N, N+1$ implies that investment in one of the assets decreases if there is an $N$ th-degree risk increase in its return rate. ${ }^{19}$

\section{REFERENCES}

Chateauneuf, Alain and Ghizlane Lakhnati (2007): "From Sure to Strong Diversification," Economic Theory, 32, 511-522.

Cheng, H., M. J. Magill, and W. Shafer. (1987): "Some Results on Comparative Statics under Uncertainty," International Economic Review, 28 (2), 493-507.

Chew, Soo Hong, Edi Karni and Zvi Safra (1987): "Risk Aversion in the Theory of Expected Utility with Rank Dependent Probabilities," Journal of Economic Theory, 42, 370-381.

Chiu, W. Henry (2018a): "An Ordinal Theory of Risk Aversion," SSRN Working paper.

Chiu, W. Henry (2018b): "An Ordinal Theory of Risk and Ambiguity Aversion," SSRN Working paper.

Chiu, W. Henry and Louis Eeckhoudt (2010): "The Effects of Stochastic Wages and Non-labor Income on Labor Supply: Update and Extensions," Journal of Economics, 100 (1), 69-83.

Chiu, W. Henry, Louis Eeckhoudt, and Beatrice Rey (2012): "On Relative and Partial Risk Attitudes: Theory and Implications," Economic Theory, 50(1), 151167

Cohen, Michele D. (1995): "Risk-Aversion Concepts in Expected and Non-Expected-Utility Models," Geneva Papers on Risk and Insurance Theory, 20, 73-91.

\footnotetext{
${ }^{19}$ In the case of $N=1$, (13) states that the "relative risk aversion" measure is less than unity, which is shown by Cheng, Magill, and Shafer (1987) to be sufficient to sign the effect of an first-degree stochastic dominant deterioration in the return of one of the assets.
} 
Debreu, Gerard (1964): "Continuity Properties of Paretian Utility," International Economic Review, 5, 285-293.

Dekel, Eddie (1989): "Asset Demands without the Independence Axiom," Econometrica, 57(1), 163-169.

Eeckhoudt, Louis and Harris Schlesinger (2006): "Putting Risk in Its Proper Place," American Economic Review, 96, 280-289.

Eeckhoudt, Louis and Harris Schlesinger (2008): "Changes in Risk and the Demand for Saving," Journal of Monetary Economics, 55 (7), 1329-1336.

Ekern, Steinar. (1980): "Increasing Nth Degree Risk," Economics Letters, 6, 329-333.

Hadar, Josef, and Tae Kun Seo. (1990): “The Effects of Shifts in a Return Distribution on Optimal Portfolios," International Economic Review, 31 (3), 721-736.

Kahneman, Daniel and Amos Tversky (1979): "Prospect Theory: an Analysis of Decision under Risk," Econometrica, 47(2), 263-291.

Kimball MS (1990) Precautionary Saving in the Small and the Large. Econometrica 58: 53-73.

Leland, Hayne E. (1968): "Saving and Uncertainty: The Precautionary Demand for Saving." Quarterly Journal of Economics, 82, 465-473.

Machina, Mark (1982a): "Expected Utility Analysis without the Independence Axiom," Econometrica 50, 277-323.

Machina, Mark (1982b): "A Strong Characterization of Decreasing Risk Aversion," Econometrica 50(4), 1069-1079.

Machina, Mark (1989): "Comparative Statics and Non-expected Utility Preferences," Journal of Economic Theory 47, 393-405.

Machina, Mark (2009): "Risk, Ambiguity, and the Rank-Dependence Axioms," American Economic Review 99(1), 385-392.

Machina, Mark and Neilson, William (1987): "The Ross Measure of Risk Aversion: Strengthening and Extension," Econometrica 55, 1139-1149. 
Machina, Mark J. and John W. Pratt (1997): "Increasing Risk: Some Direct Constructions, " Journal of Risk and Uncertainty, 14, 103-127.

Menezes, C. F., C. Geiss, and J. Tressler. (1980): "Increasing Downside Risk," American Economic Review, 921-932.

Rothschild, Michael and Joseph Stiglitz (1970): "Increasing risk I: A definition," Journal of Economic Theory, 2, 225-243.

Rothschild, Michael and Joseph Stiglitz (1971): "Increasing risk II: Its Economic Consequences", Journal of Economic Theory, 2, 225-243.

Starmer, Chris (2000): "Developments in Non-expected Utility Theory: The Hunt for a Descriptive Theory of Choice under Risk," Journal of Economic Literature, 38(2), 332-382.

Tobin, James (1958): "Liquidity Preference as behavior towards Risk," Review of Economic Studies, 25, 65-86.

Wang, T. (1993) $L_{p}$-Fréchet Differentiable Preference and Local Utility Analysis, Journal of Economic Theory, 61, 139-59.

Wright, Randall (1987): "Expectation Dependence of Random Variables, with an Application in Portfolio Theory," Theory and Decision, 22, 111-124.

Wu, George (1994): "An Empirical Test of Ordinal Independence," Journal of Risk and Uncertainty, 9, 39-60. 